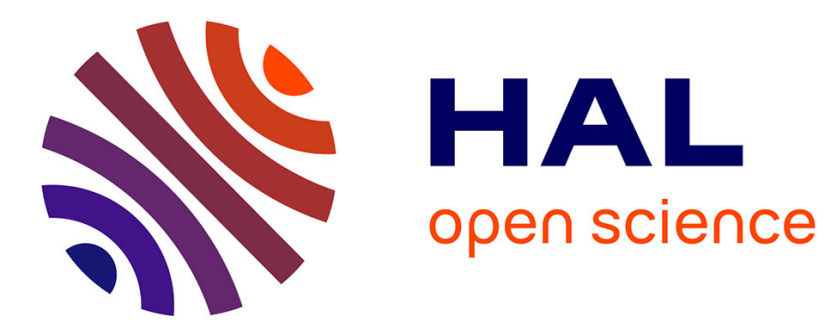

\title{
Discrete Optimization of Robin Transmission Conditions for Anisotropic Diffusion with Discrete Duality Finite Volume Methods
}

\author{
Martin J. Gander, Laurence Halpern, Florence Hubert, Stella Krell
}

\section{- To cite this version:}

Martin J. Gander, Laurence Halpern, Florence Hubert, Stella Krell. Discrete Optimization of Robin Transmission Conditions for Anisotropic Diffusion with Discrete Duality Finite Volume Methods. Vietnam Journal of Mathematics, 2021, 10.1007/s10013-021-00518-3 . hal-02539124v2

HAL Id: hal-02539124

https://hal.science/hal-02539124v2

Submitted on 14 May 2021

HAL is a multi-disciplinary open access archive for the deposit and dissemination of scientific research documents, whether they are published or not. The documents may come from teaching and research institutions in France or abroad, or from public or private research centers.
L'archive ouverte pluridisciplinaire HAL, est destinée au dépôt et à la diffusion de documents scientifiques de niveau recherche, publiés ou non, émanant des établissements d'enseignement et de recherche français ou étrangers, des laboratoires publics ou privés. 


\title{
Discrete Optimization of Robin Transmission Conditions for Anisotropic Diffusion with Discrete Duality Finite Volume Methods
}

\author{
Martin J. Gander * $\quad$ Laurence Halpern ${ }^{\dagger} \quad$ Florence Hubert ${ }^{\ddagger} \quad$ Stella Krell ${ }^{\S}$
}

April 19, 2021

\begin{abstract}
Discrete Duality Finite Volume (DDFV) methods are very well suited to discretize anisotropic diffusion problems, even on meshes with low mesh quality. Their performance stems from an accurate reconstruction of the gradients between mesh cell boundaries, which comes however at the cost of using both a primal (cell centered) and a dual (vertex centered) mesh, and thus leads to larger system sizes. To solve these systems, we propose to use non-overlapping optimized Schwarz methods with Robin transmission conditions, which can also well take into account anisotropic diffusion across subdomain interfaces. We study these methods here directly at the discrete level, and prove convergence using energy estimates for general decompositions including cross points and fully anisotropic diffusion. Our analysis reveals that primal and dual meshes might be coupled using different optimized Robin parameters in the optimized Schwarz methods. We present both the separate and coupled optimization of Robin transmission conditions and derive parameters which lead to the fastest possible convergence in each case. We illustrate our results with numerical experiments for the model problem, and also in situations that go beyond our analysis, with an application to anisotropic image reconstruction.
\end{abstract}

\section{Introduction}

Anisotropic diffusion problems arise in many applications, from geology [31] to medicine [11], but more recently they play a major role in image reconstruction [32], pioneered by the Perona-Malik non-linear partial differential equation [30], see also the fundamental contributions of the Slovak school [25, 29, 10]. With the ever increasing demand for high accuracy and rapid solution, and the availability of more and more highly parallel computing systems, parallel algorithms to simulate such problems are in high demand, in particular domain decomposition methods which are naturally parallel. Non-overlapping optimized Schwarz methods form a class of such domain decomposition methods; for an introduction, see $[12,13]$ and references therein. They were introduced for anisotropic diffusion problems at the continuous level in [17], where their convergence was proved for a two subdomain decomposition using energy estimates, and optimized transmission conditions between the subdomains were derived, also at the continuous level. For a reduction of the anisotropic optimization of the transmission conditions to an isotropic one, see [15, Section 5]. To use such algorithms on a computer, they have to be discretized, and for anisotropic diffusion problems, discrete duality finite volume (DDFV) methods are well suited because of their accurate gradient reconstruction. This has been beneficial in many situations: see [23, 3] for diffusion problems with discontinuous coefficients, [6] for div-curl, [5] for advection-diffusion, [7] for Stokes, and [24] for Maxwell problems, and [21] for image reconstruction. A first variant of a DDFV optimized Schwarz algorithm for fluid mechanics can be found in [26, 4]; its convergence did however

\footnotetext{
*University of Geneva, 2-4 rue du Lièvre, CP 64, 1211 Genève, Switzerland martin.gander@unige.ch

†LAGA, Université Sorbonne Paris Nord, 93430 Villetaneuse, FRANCE halpern@math.univ-paris13.fr

¥I2M, Aix-Marseille Université, 39 rue F. Joliot Curie, 13453 Marseille, Cedex 13, FRANCEflorence.hubert@univ-amu.fr
§Université Côte d'Azur, CNRS, Inria, LJAD, France stella.krell@univ-cotedazur.fr
} 
not reach the full potential of optimized Schwarz methods, due to the discretization technique used at the interfaces. An improved treatment of the transmission conditions can be found in $[18,16]$, see also [2] for a space-time variant, and a first application to anisotropic diffusion was tested in [17]. There has however so far never been a convergence analysis of DDFV optimized Schwarz methods at the discrete level for general decompositions, including cross points, and also the optimization of the transmission conditions at the discrete level is lacking. In [17], it was discovered that the best working parameters in the transmission conditions can differ substantially from the ones predicted by the continuous analysis for anisotropic diffusion, and a similar discovery was made in $[33,34]$ for various discretizations of evolution problems, see also [22]. We therefore study here for the first time non-overlapping optimized Schwarz methods with Robin transmission conditions at the discrete level for DDFV discretizations of anisotropic diffusion problems. We give a convergence proof using energy estimates for general decompositions into many subdomains including cross points, and then optimize the transmission conditions at the discrete level. Our analysis reveals an interesting, new interplay between the primal and dual mesh components of the DDFV discretization and the optimized transmission conditions used in optimized Schwarz methods, a feature which remained hidden in the continuous analysis in [17]. We derive optimized transmission conditions, both when using separate parameters on the primal and dual mesh, or the same for both. We then compare our discrete optimized transmission conditions to the ones obtained at the continuous level in [17], and illustrate our findings using numerical experiments, both for model problems and discretizations covered and not covered by our analysis, and an example in image reconstruction that goes quite beyond, mixing different mesh types and containing cross points, with an application in image reconstruction.

\section{Optimized Schwarz for Anisotropic Diffusion}

We are interested in the solution of anisotropic diffusion problems of the form

$$
\begin{array}{rlll}
\mathcal{L}(u):=-\operatorname{div}(A \nabla u)+\eta u & = & & \text { in } \Omega, \\
u & = & 0 & \text { on } \partial \Omega,
\end{array}
$$

where $A$ is a symmetric positive definite matrix with $L^{\infty}$ coefficients,

$$
(x, y) \in \Omega \mapsto A(x, y)=\left(\begin{array}{ll}
A_{x x}(x, y) & A_{x y}(x, y) \\
A_{x y}(x, y) & A_{y y}(x, y)
\end{array}\right),
$$

and $(x, y) \in \Omega \mapsto \eta(x, y) \geq 0$ is a given non-negative function in $L^{\infty}$. A DDFV discretization on arbitrary domains and meshes of the anisotropic diffusion problem (2.1), and the associated non-overlapping optimized Schwarz solver were introduced in [17], and convergence of the Schwarz method was proved at the discrete level for a two subdomain decomposition using energy estimates. We are interested here in a complete discrete convergence analysis of the optimized Schwarz method with Robin transmission conditions for general decompositions including cross points, and also an optimization of the discrete convergence factor, since differences between the continuous analysis and discrete performance were pointed out in [17].

\subsection{Classical notation for DDFV schemes}

DDFV discretizations need a certain amount of notation for which we follow [1]. A DDFV mesh $\mathcal{T}$ consists of a primal mesh $\mathfrak{M}$, the black triangles in the example in Figure 1 on the left, leading to a cell centered (CC) scheme, and a dual mesh $\mathfrak{M}^{*} \cup \partial \mathfrak{M}^{*}$, for which we show only two light red cells in Figure 1 on the left, leading to a vertex centered (VC) scheme. The primal mesh $\mathfrak{M}$ is a set of disjoint open polygonal control volumes $\mathrm{K} \subset \Omega$ such that $\cup \overline{\mathrm{K}}=\bar{\Omega}$. We denote by $\partial \mathfrak{M}$ the set of edges of the control volumes in $\mathfrak{M}$ included in $\partial \Omega$, which we consider as degenerate control volumes. For all neighboring control volumes $\mathrm{K}$ and $\mathrm{L}$, we assume that $\partial \mathrm{K} \cap \partial \mathrm{L}$ is an edge of the primal mesh denoted by $\sigma=\mathrm{K} \mid \mathrm{L}$. To each control volume and degenerate control volume $\mathrm{K} \in \mathfrak{M} \cup \partial \mathfrak{M}$, we associate a point $x_{\mathrm{K}} \in \mathrm{K}$, see the black dots in Figure 1. This family of points is denoted by $X=\left\{x_{\mathrm{K}}, \mathrm{K} \in \mathfrak{M} \cup \partial \mathfrak{M}\right\}$. Let $X^{*}$ denote the set of vertices $x_{\mathrm{K}^{*}}$ of the primal control volumes in $\mathfrak{M}$, see the red squares in Figure 1 . We split this set into $X^{*}=X_{i n t}^{*} \cup X_{b n d}^{*}$ where $X_{\text {int }}^{*} \cap \partial \Omega=\emptyset$ (filled red squares) and $X_{b n d}^{*} \subset \partial \Omega$ (not filled red squares). 


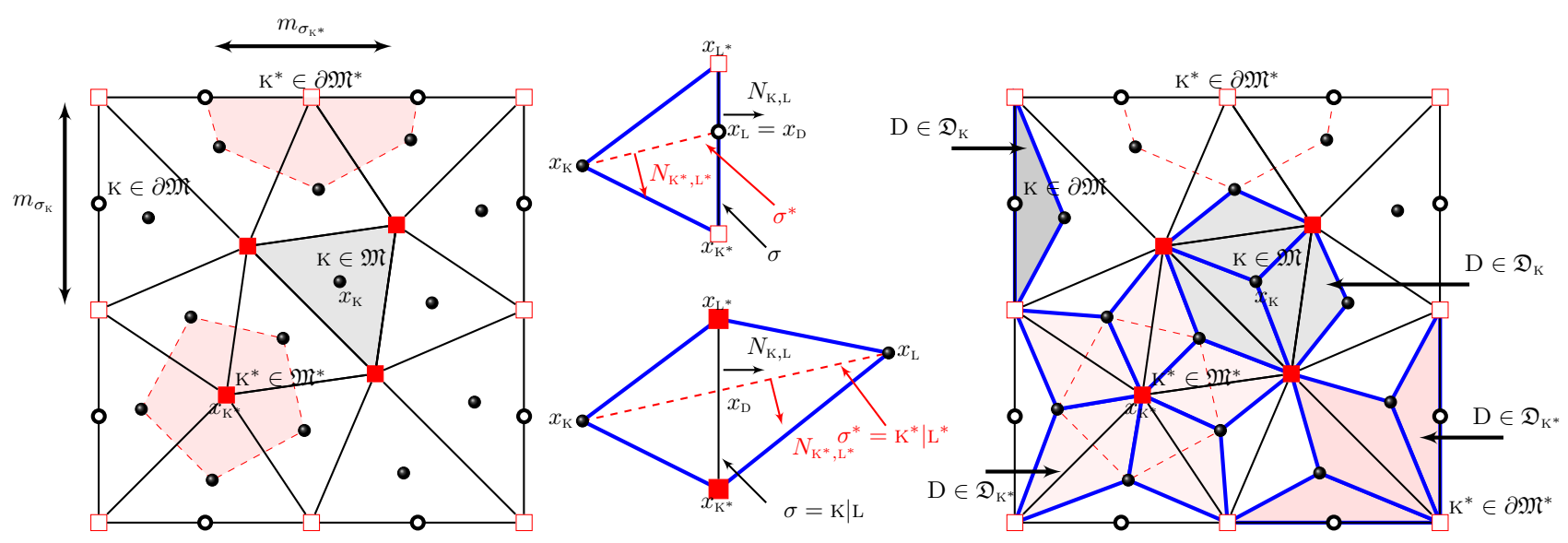

Figure 1: DDFV notation. Left: primal mesh and example of an interior and a boundary dual cell. Middle top: example of a boundary diamond cell. Middle bottom: example of an interior diamond cell. Right: example of diamond cells associated with $x_{\mathrm{K}}$ and $x_{\mathrm{K}^{*}}$ in the interior and on the boundary.

For each $x_{\mathrm{K}^{*}}$ we also introduce a control volume, see the light red polygons in Figure 1 on the left for an interior and boundary control volume example, whose corners are the $x_{\mathrm{K}}$. All these control volumes define the set $\mathfrak{M}^{*} \cup \partial \mathfrak{M}^{*}$ of dual control volumes that forms also a partition of $\Omega$ into disjoint polygonal control volumes.

Given the sets $X$ and $X^{*}$, we define the diamond cells $\mathrm{D}_{\sigma, \sigma^{*}}$ being the quadrangles whose diagonals are a primal edge $\sigma=\mathrm{K} \mid \mathrm{L}=\left(x_{\mathrm{K}^{*}}, x_{\mathrm{L}^{*}}\right)$ and a dual edge $\sigma^{*}=\mathrm{K} \mid \mathrm{L}=\left(x_{\mathrm{K}}, x_{\mathrm{L}}\right)$, see Figure 1 in the middle for two examples. The set of diamond cells is denoted by $\mathfrak{D}$, and it has two disjoint subsets: the interior diamond cells $\mathrm{D} \in \mathfrak{D}_{\text {int }}$ (e.g. Figure 1 middle bottom), and the boundary diamond cells $\mathrm{D} \in \mathfrak{D}_{\text {bnd }}(\mathrm{e} . \mathrm{g}$. Figure 1 middle top) for which $\left[x_{\mathrm{K}^{*}}, x_{\mathrm{L}^{*}}\right] \subset \partial \Omega$, where $x_{\mathrm{L}} \in\left(x_{\mathrm{K}^{*}}, x_{\mathrm{L}^{*}}\right)$. The complete domain is also formed by all the diamonds, $\bar{\Omega}=\cup_{\mathrm{D} \in \mathfrak{D}} \overline{\mathrm{D}}$. To each diamond $\mathrm{D} \in \mathfrak{D}$, we associate a point $x_{\mathrm{D}} \in\left[x_{\mathrm{K}^{*}}, x_{\mathrm{L}^{*}}\right]$. Let $\mathfrak{D}_{\mathrm{K}}$ be the set of diamonds with $x_{\mathrm{K}}$ as vertex and $\mathfrak{D}_{\mathrm{K}^{*}}$ be the set of diamonds with $x_{\mathrm{K}^{*}}$ as vertex, see Figure 1 on the right for examples.

For any primal control volume $\mathrm{K} \in \mathfrak{M} \cup \partial \mathfrak{M}$, we denote by $m_{\mathrm{K}}$ its Lebesgue measure, and similarly use $m_{\mathrm{K}^{*}}$ for the dual control volumes. For a diamond cell $\mathrm{D}$ with vertices $\left(x_{\mathrm{K}}, x_{\mathrm{K}^{*}}, x_{\mathrm{L}}, x_{\mathrm{L}^{*}}\right)$, we denote by $x_{\mathrm{D}}$ the center of the diamond cell $\mathrm{D}$, that is the intersection of the primal edge $\sigma$ and the dual edge $\sigma^{*}$, $m_{\mathrm{D}}$ its measure, $m_{\sigma}$ the length of the primal edge $\sigma, m_{\sigma^{*}}$ the length of the dual edge $\sigma^{*}$, and $m_{\sigma_{\mathrm{K}^{*}}}$ the measure of $\partial \mathrm{K}^{*} \cap \partial \Omega$.

In DDFV, a variable $u_{\mathrm{K}}$ is associated with all primal control volumes $\mathrm{K} \in \mathfrak{M} \cup \partial \mathfrak{M}$, and a variable $u_{\mathrm{K}^{*}}$ is associated with all dual control volumes $\mathrm{K}^{*} \in \mathfrak{M}^{*} \cup \partial \mathfrak{M}^{*}$. We denote the approximate solution on the mesh $\mathcal{T}$ by $u_{\mathcal{T}} \in \mathbb{R}^{\mathcal{T}}$, where

$$
u_{\mathcal{T}}:=\left(\left(u_{\mathrm{K}}\right)_{\mathrm{K} \in(\mathfrak{M} \cup \partial \mathfrak{M})},\left(u_{\mathrm{K}^{*}}\right)_{\mathrm{K}^{*} \in\left(\mathfrak{M}^{*} \cup \partial \mathfrak{M} *\right)}\right)
$$

Following $[23,9]$, we define a consistent approximation of the gradient operator denoted by $\nabla^{\mathfrak{D}}: u_{\mathcal{T}} \in$ $\mathbb{R}^{\mathcal{T}} \mapsto\left(\nabla^{\mathrm{D}} u_{\mathcal{T}}\right)_{\mathrm{D} \in \mathfrak{D}} \in\left(\mathbb{R}^{2}\right)^{\mathfrak{D}}$ by

$$
\nabla^{\mathrm{D}} u_{\mathcal{T}}:=\frac{1}{2 m_{\mathrm{D}}}\left[\left(u_{\mathrm{L}}-u_{\mathrm{K}}\right) \mathbf{N}_{\mathrm{KL}}+\left(u_{\mathrm{L}^{*}}-u_{\mathrm{K}^{*}}\right) \mathbf{N}_{\mathrm{K}^{*} \mathrm{~L}^{*}}\right], \quad \forall \mathrm{D} \in \mathfrak{D},
$$

with $\mathbf{N}_{\mathrm{KL}}:=\left(x_{\mathrm{L}^{*}}-x_{\mathrm{K}^{*}}\right)^{\perp}$ and $\mathbf{N}_{\mathrm{K}^{*} \mathrm{~L}^{*}}:=\left(x_{\mathrm{L}}-x_{\mathrm{K}}\right)^{\perp}$, where for any vector $(x, y),(x, y)^{\perp}:=(-y, x)$. We also define a consistent approximation of the divergence operator denoted by $\operatorname{div}^{\mathcal{T}}: \xi_{\mathfrak{D}}=\left(\xi_{\mathrm{D}}\right)_{\mathrm{D} \in \mathfrak{D}} \in$ 
$\left(\mathbb{R}^{2}\right)^{\mathfrak{D}} \mapsto \operatorname{div}^{\mathcal{T}} \xi_{\mathfrak{D}} \in \mathbb{R}^{\mathcal{T}}$ by

$$
\begin{aligned}
\operatorname{div}^{\mathrm{K}} \xi_{\mathfrak{D}} & :=\frac{1}{m_{\mathrm{K}}} \sum_{\mathrm{D} \in \mathfrak{D}_{\mathrm{K}}}\left(\xi_{\mathrm{D}}, \mathbf{N}_{\mathrm{KL}}\right), \forall \mathrm{K} \in \mathfrak{M}, \quad \text { and } \operatorname{div}^{\mathrm{K}} \xi_{\mathfrak{D}}=0, \forall \mathrm{K} \in \partial \mathfrak{M}, \\
\operatorname{div}^{\mathrm{K}^{*}} \xi_{\mathfrak{D}} & :=\frac{1}{m_{\mathrm{K}^{*}}} \sum_{\mathrm{D} \in \mathfrak{D}_{\mathrm{K}^{*}}}\left(\xi_{\mathrm{D}}, \mathbf{N}_{\mathrm{K}^{*} \mathrm{~L}^{*}}\right), \forall \mathrm{K}^{*} \in \mathfrak{M}^{*} \cup \partial \mathfrak{M}^{*}
\end{aligned}
$$

For $u_{\mathcal{T}} \in \mathbb{R}^{\mathcal{T}}$ and $f_{\mathcal{T}} \in \mathbb{R}^{\mathcal{T}}$, we denote by $\mathcal{L}^{\mathcal{T}}\left(u_{\mathcal{T}}, f_{\mathcal{T}}\right)=0$ the linear system

$$
\begin{aligned}
& -\operatorname{div}^{\mathrm{K}}\left(A_{\mathfrak{D}} \nabla^{\mathfrak{D}} u_{\mathcal{T}}\right)+\eta_{\mathrm{K}} u_{\mathrm{K}}=f_{\mathrm{K}}, \quad \forall \mathrm{K} \in \mathfrak{M}, \\
& -\operatorname{div}^{\mathrm{K}^{*}}\left(A_{\mathfrak{D}} \nabla^{\mathfrak{D}} u_{\mathcal{T}}\right)+\eta_{\mathrm{K}^{*}} u_{\mathrm{K}^{*}}=f_{\mathrm{K}^{*}}, \quad \forall \mathrm{K}^{*} \in \mathfrak{M}^{*}, \\
& u_{\mathrm{K}}=0, \quad \forall \mathrm{K} \in \partial \mathfrak{M}, \quad u_{\mathrm{K}^{*}}=0, \quad \forall \mathrm{K}^{*} \in \partial \mathfrak{M}^{*} \text {, }
\end{aligned}
$$

where for smooth functions $A, f$ and $\eta$ we use pointwise evaluations

$$
\begin{aligned}
A_{\mathfrak{D}} & :=\left(A_{\mathrm{D}}\right)_{\mathrm{D} \in \mathfrak{D}}, \quad A_{\mathrm{D}}:=A\left(x_{\mathrm{D}}\right), \\
f_{\mathcal{T}} & :=\left(\left(f_{\mathrm{K}}\right)_{\mathrm{K} \in(\mathfrak{M} \cup \partial \mathfrak{M})},\left(f_{\mathrm{K}^{*}}\right)_{\mathrm{K}^{*} \in\left(\mathfrak{M}^{*} \cup \partial \mathfrak{M}^{*}\right)}\right), \quad f_{\mathrm{K}}:=f\left(x_{\mathrm{K}}\right), \quad f_{\mathrm{K}^{*}}:=f\left(x_{\mathrm{K}^{*}}\right), \\
\eta_{\mathcal{T}} & :=\left(\left(\eta_{\mathrm{K}}\right)_{\mathrm{K} \in(\mathfrak{M} \cup \partial \mathfrak{M})},\left(\eta_{\mathrm{K}^{*}}\right)_{\mathrm{K}^{*} \in\left(\mathfrak{M}^{*} \cup \partial \mathfrak{M}^{*}\right)}\right), \quad \eta_{\mathrm{K}}:=\eta\left(x_{\mathrm{K}}\right), \quad \eta_{\mathrm{K}^{*}}:=\eta\left(x_{\mathrm{K}^{*}}\right) .
\end{aligned}
$$

For non-smooth functions, mean values of the functions can be used. We also recall the fundamental discrete duality property satisfied by these operators,

$$
\left(\operatorname{div}^{\mathcal{T}} \xi_{\mathfrak{D}}, u_{\mathcal{T}}\right)_{\mathcal{T}}:=\frac{1}{2}\left(\sum_{\mathrm{K} \in \mathfrak{M}} m_{\mathrm{K}} \operatorname{div}^{\mathrm{K}} \xi_{\mathfrak{D}} u_{\mathrm{K}}+\sum_{\mathrm{K}^{*} \in \mathfrak{M}^{*}} m_{\mathrm{K}^{*}} \operatorname{div}^{\mathrm{K}^{*}} \xi_{\mathfrak{D}} u_{\mathrm{K}^{*}}\right)=-\sum_{\mathrm{D} \in \mathfrak{D}} m_{\mathrm{D}}\left(\xi_{\mathrm{D}}, \nabla^{\mathrm{D}} u_{\mathcal{T}}\right):=\left(\xi_{\mathfrak{D}}, \nabla^{\mathfrak{D}} u_{\mathcal{T}}\right)_{\mathfrak{D}}
$$

\subsection{DDFV on Composite Meshes}

In the case of a general domain decomposition into many subdomains $\Omega=\cup_{j=1, \cdots, J} \Omega_{j}$ including cross points, we consider for each subdomain $\Omega_{j}$ a DDFV mesh $\mathcal{T}_{j}=\left(\mathfrak{M}_{j} \cup \partial \mathfrak{M}_{j}, \mathfrak{M}_{j}^{*} \cup \partial \mathfrak{M}_{j}^{*}\right)$, and the associated diamond mesh $\mathfrak{D}_{j}$. We assume that $\Omega_{j}$ is covered by the primal mesh $\mathfrak{M}_{j}$, so that $\mathfrak{M}=\cup \mathfrak{M}_{j}$ can be taken as the primal mesh of a DDFV mesh $\mathcal{T}$ associated to $\Omega$. This induces that the interface $\Gamma_{j i}$ between the two sub-domains $\Omega_{j}$ and $\Omega_{i}$ is covered by primal boundary edges of $\partial \mathfrak{M}_{j}$ or $\partial \mathfrak{M}_{i}$, and that corner points are located at the centers of their dual cells.

For $\Omega_{j}$, we denote the set of neighboring indices by $I_{j}:=\left\{i\right.$ such that $\left.\Gamma_{j i} \neq \emptyset\right\}$ where $\Gamma_{j i}$ is the interface between $\Omega_{j}$ and $\Omega_{i}$. We denote by

$$
\begin{aligned}
& \mathfrak{D}_{j, \Gamma_{i}}:=\left\{\mathrm{D} \in \mathfrak{D}_{j}, \mathrm{D} \cap \Gamma_{j i} \neq \emptyset\right\} \text { the diamond cells intersecting } \Gamma_{j i}, \\
& \partial \mathfrak{M}_{j, \Gamma_{i}}:=\left\{\mathrm{K} \in \partial \mathfrak{M}_{j}, \mathrm{~K} \cap \Gamma_{j i} \neq \emptyset\right\} \text { the boundary primal cells intersecting } \Gamma_{j i}, \\
& \partial \mathfrak{M}_{j, \Gamma_{i}, i n t}^{*}:=\left\{\mathrm{K}^{*} \in \partial \mathfrak{M}_{j}^{*}, \mathrm{~K}^{*} \cap \Gamma_{j i} \neq \emptyset\right\} \text { the interior boundary dual cells intersecting } \Gamma_{j i}, \\
& \partial \mathfrak{M}_{j, D}:=\left\{\mathrm{K} \in \partial \mathfrak{M}_{j}, \mathrm{~K} \cap \partial \Omega \neq \emptyset\right\} \text { the boundary primal cells intersecting } \partial \Omega, \\
& \partial \mathfrak{M}_{j, D}^{*}:=\left\{\mathrm{K}^{*} \in \partial \mathfrak{M}_{j}^{*}, \mathrm{~K}^{*} \cap \partial \Omega \neq \emptyset\right\} \text { the boundary dual cells intersecting } \partial \Omega .
\end{aligned}
$$

We call the set of all interface diamond cells

$$
\mathfrak{D}_{j, \Gamma}:=\cup_{i \in I_{j}} \mathfrak{D}_{j, \Gamma_{i}}
$$

The set of all boundary primal cells not located on a Dirichlet boundary is called

$$
\partial \mathfrak{M}_{j, \Gamma}:=\cup_{i \in I_{j}} \partial \mathfrak{M}_{j, \Gamma_{i}}
$$

The set of all boundary dual cells not located on a Dirichlet boundary is called

$$
\partial \mathfrak{M}_{j, \Gamma, i n t}^{*}:=\cup_{i \in I_{j}} \partial \mathfrak{M}_{j, \Gamma_{i}, i n t}^{*} .
$$



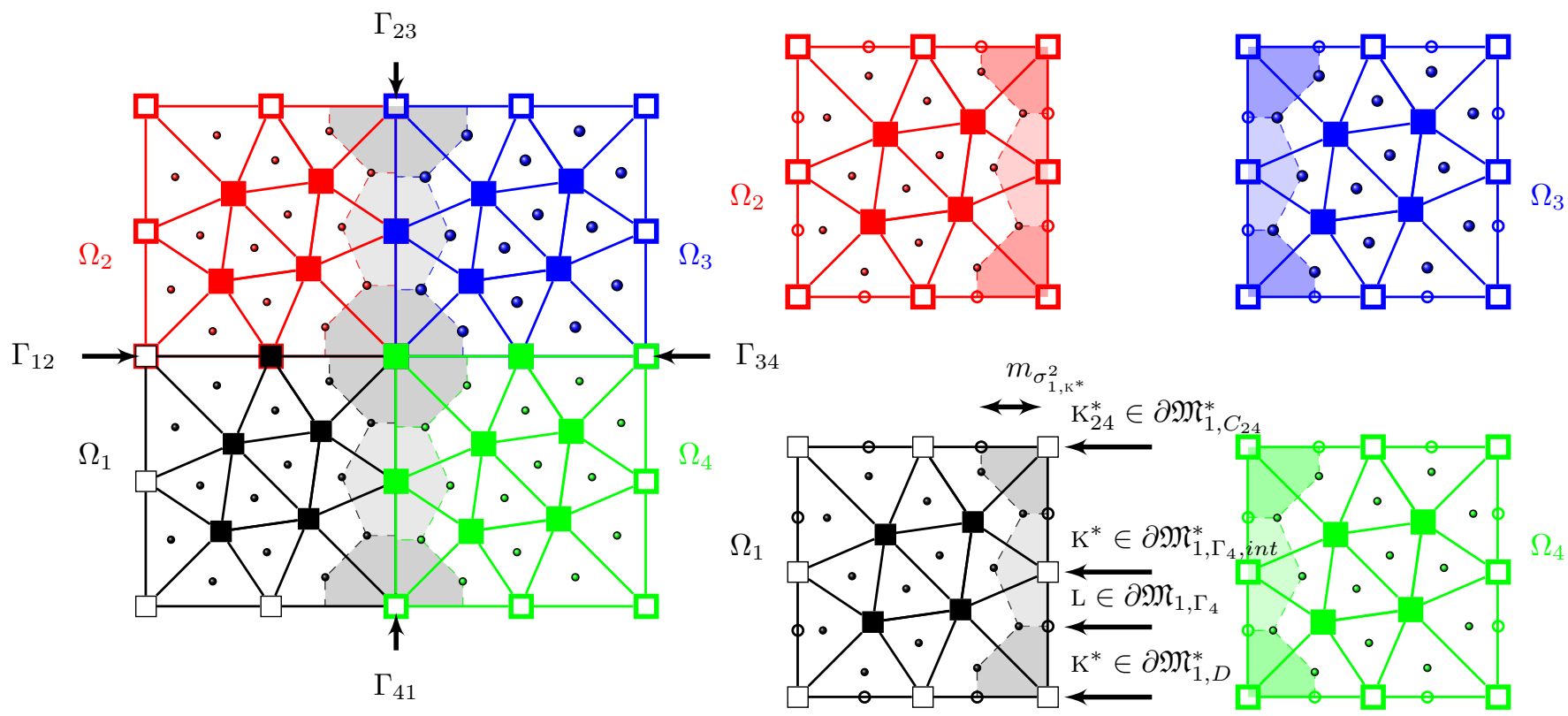

Figure 2: Left: example of a mesh for $\Omega$, with dual cells crossing $\Gamma_{23}$ and $\Gamma_{41}$. Right: example of a composite mesh associated to the domain decomposition $\Omega=\cup_{i=1}^{4} \Omega_{i}$ on the left, with the notation for the interface $\Gamma_{41}$ with boundary primal and dual cells.

Finally, at cross points $C_{i k}$, i.e. for $i, k \in I_{j}$ such that $\Gamma_{j i} \cap \Gamma_{j k}=\left\{C_{i k}\right\}$, see Figure 2,

let

$$
\partial \mathfrak{M}_{j, C_{i k}}^{*}:=\left\{\mathrm{K}^{*} \in \partial \mathfrak{M}_{j}^{*}, x_{\mathrm{K}^{*}}=C_{i k}\right\}
$$

denote the dual cross point cells, and we define the cross point and interface sets

$$
\partial \mathfrak{M}_{j, C}^{*}:=\cup_{i, k \in I_{j}} \partial \mathfrak{M}_{j, C_{i k}}^{*}, \quad \partial \mathfrak{M}_{j, \Gamma}^{*}=\partial \mathfrak{M}_{j, \Gamma, \text { int }}^{*} \cup \partial \mathfrak{M}_{j, C}^{*} .
$$

We assume that the meshes $\mathcal{T}_{j}$ are compatible in the following sense:

1. If $i \in I_{j}$, then the two meshes $\mathcal{T}_{j}$ and $\mathcal{T}_{i}$ have the same vertices on $\Gamma_{j i}$. This implies in particular that the two meshes have the same degenerate control volumes on $\Gamma_{j i}$, that is $\partial \mathfrak{M}_{j, \Gamma_{i}}=\partial \mathfrak{M}_{i, \Gamma_{j}}$.

2. The edges $\sigma$, whose center is denoted by $x_{\mathrm{L}}$, can be assimilated to a primal degenerated boundary control volume for both meshes, i.e. $\mathrm{L} \subset \partial \mathfrak{M}_{j} \cap \partial \mathfrak{M}_{i}$.

We next define the DDFV discretization for the transmission conditions of Robin type. We associate

- one unknown per interior and boundary primal and dual cell $u_{\mathcal{T}_{j}} \in \mathbb{R}^{\mathcal{T}_{j}}$,

- one flux unknown $\psi_{\mathrm{K}^{*}}=\psi_{j, \mathrm{~K}^{*}}^{i}$ per interface interior dual cell $\mathrm{K}^{*} \in \partial \mathfrak{M}_{j, \Gamma_{i}, \text { int }}^{*}$,

- two flux unknowns $\psi_{j, \mathrm{~K}^{*}}^{i}$ and $\psi_{j, \mathrm{~K}^{*}}^{k}$ per cross point dual cell $\mathrm{K}^{*} \in \partial \mathfrak{M}_{j, C_{i k}}^{*}$.

We denote by $\psi_{\mathcal{T}_{j}} \in \mathbb{R}^{\partial \mathfrak{M}_{j, \Gamma}^{*}}$ the collection of all flux unknowns $\psi_{\mathrm{K}^{*}}$ for inner dual interface cells. Given $f_{\mathcal{T}_{j}} \in \mathbb{R}^{\mathfrak{M}_{j} \cup \mathfrak{M}_{j}^{*} \cup \partial \mathfrak{M}_{j, \Gamma}^{*}}, h_{\mathcal{T}_{j}} \in \mathbb{R}^{\partial \mathfrak{M}_{j, \Gamma} \cup \partial \mathfrak{M}_{j, \Gamma}^{*}}$ and $p, p^{*}$ two positive constants, we denote by 
$\mathcal{L}^{\mathcal{T}_{j}}\left(u_{\mathcal{T}_{j}}, \psi_{\mathcal{T}_{j}}, f_{\mathcal{T}_{j}}, h_{\mathcal{T}_{j}}\right)=0$ the linear system for $u_{\mathcal{T}_{j}} \in \mathbb{R}^{\mathcal{T}_{j}}, \psi_{\mathcal{T}_{j}} \in \mathbb{R}^{\partial \mathfrak{M}_{j, \Gamma}^{*}}$ given by

$$
\begin{aligned}
& -\operatorname{div}^{\mathrm{K}}\left(A_{\mathcal{D}} \nabla^{\mathfrak{D}} u_{\mathcal{T}_{j}}\right)+\eta_{\mathrm{K}} u_{\mathrm{K}}=f_{\mathrm{K}}, \quad \forall \mathrm{K} \in \mathfrak{M}_{j}, \\
& -\operatorname{div}^{\mathrm{K}^{*}}\left(A_{\mathfrak{D}} \nabla^{\mathcal{D}} u_{\mathcal{T}_{j}}\right)+\eta_{\mathrm{K}^{*}} u_{\mathrm{K}^{*}}=f_{\mathrm{K}^{*}}, \quad \forall \mathrm{K}^{*} \in \mathfrak{M}_{j}^{*}, \\
& -\frac{1}{m_{\mathrm{K}^{*}}}\left(\sum_{\mathrm{D} \in \mathfrak{D}_{\mathrm{K}^{*}}}\left(A_{\mathrm{D}} \nabla^{\mathrm{D}} u_{\mathcal{T}_{j}}, \mathbf{N}_{\mathrm{K}^{*} \mathrm{~L}^{*}}\right)+m_{\sigma_{\mathrm{K}^{*}}} \psi_{\mathrm{K}^{*}}\right)+\eta_{\mathrm{K}^{*}} u_{\mathrm{K}^{*}}=f_{\mathrm{K}^{*}}, \quad \forall \mathrm{K}^{*} \in \partial \mathfrak{M}_{j, \Gamma, i n t}^{*}, \\
& -\frac{1}{m_{\mathrm{K}^{*}}}\left(\sum_{\mathrm{D} \in \mathfrak{D}_{\mathrm{K}^{*}}}\left(A_{\mathrm{D}} \nabla^{\mathrm{D}} u_{\mathcal{T}_{j}}, \mathbf{N}_{\mathrm{K}^{*} \mathrm{~L}^{*}}\right)+m_{\sigma_{j, \mathrm{~K}^{*}}^{i}} \psi_{j, \mathrm{~K}^{*}}^{i}+m_{\sigma_{j, \mathrm{~K}^{*}}^{k}} \psi_{j, \mathrm{~K}^{*}}^{k}\right)+\eta_{\mathrm{K}^{*}} u_{\mathrm{K}^{*}}=f_{\mathrm{K}^{*}}, \quad \forall \mathrm{K}^{*} \in \partial \mathfrak{M}_{j, C_{i k}}^{*}, \\
& \frac{1}{m_{\sigma}}\left(A_{\mathrm{D}} \nabla^{\mathrm{D}} u_{\mathcal{\tau}_{j}}, \mathbf{N}_{\mathrm{KL}}\right)+p u_{\mathrm{L}}=h_{j, \mathrm{~L}}, \quad \forall \mathrm{L} \in \partial \mathfrak{M}_{j, \Gamma}, \\
& \psi_{\mathrm{K}^{*}}+p^{*} u_{\mathrm{K}^{*}}=h_{j, \mathrm{~K}^{*}}, \forall \mathrm{K}^{*} \in \partial \mathfrak{M}_{j, \Gamma, \text { int }}^{*}, \\
& \psi_{j, \mathrm{~K}^{*}}^{k}+p^{*} u_{\mathrm{K}^{*}}=h_{j, \mathrm{~K}^{*}}^{k} \text { and } \psi_{j, \mathrm{~K}^{*}}^{i}+p^{*} u_{\mathrm{K}^{*}}=h_{j, \mathrm{~K}^{*}}^{i}, \forall \mathrm{K}^{*} \in \partial \mathfrak{M}_{j, C_{i k}}^{*}, \\
& u_{\mathrm{K}}=0, \forall \mathrm{K} \in \partial \mathfrak{M}_{j, D}, \quad u_{\mathrm{K}^{*}}=0, \quad \forall \mathrm{K}^{*} \in \partial \mathfrak{M}_{j, D}^{*},
\end{aligned}
$$

where we added the subscript $j$ in the interface data $h_{j}$ to denote this is data for subdomain $\Omega_{j}$. Equations (2.7a)-(2.7d) correspond to an approximation of the equation after integration on $\mathfrak{M}_{j}, \mathfrak{M}_{j}^{*}$ and $\partial \mathfrak{M}_{j}^{*}$. Equations $(2.7 \mathrm{e})-(2.7 \mathrm{~g})$ are related to the Robin transmission conditions on $\partial \mathfrak{M}_{j, \Gamma}$ and $\partial \mathfrak{M}_{j, \Gamma}^{*}$. Finally, equation $(2.7 \mathrm{~h})$ corresponds to the homogeneous Dirichlet boundary condition on $\partial \Omega$.

Theorem 2.1 (Well-posedness of the DDFV subdomain problems). For any $f_{\mathcal{T}_{j}} \in \mathbb{R}^{\mathfrak{M}_{j} \cup \mathfrak{M}_{j}^{*} \cup \partial \mathfrak{M}_{j, \Gamma}^{*}}$ and $h_{\mathcal{T}_{j}} \in \mathbb{R}^{\partial \mathfrak{M}_{j, \Gamma} \cup \partial \mathfrak{M}_{j, \Gamma}^{*}}$, there exists a unique solution $\left(u_{\mathcal{T}_{j}}, \psi_{\mathcal{T}_{j}}\right) \in \mathbb{R}^{\mathcal{T}_{j}} \times \mathbb{R}^{\partial \mathfrak{M}_{j, \Gamma} \cup \partial \mathfrak{M}_{j, \Gamma}^{*}}$ of the linear system

$$
\mathcal{L}^{\mathcal{T}_{j}}\left(u_{\mathcal{T}_{j}}, \psi_{\mathcal{T}_{j}}, f_{\mathcal{T}_{j}}, h_{\mathcal{T}_{j}}\right)=0 .
$$

Proof. By linearity, since the number of unknowns and the number of equations coincide, it is sufficient to prove that if $\mathcal{L}^{\mathcal{T}_{j}}\left(u_{\mathcal{T}_{j}}, \psi_{\mathcal{T}_{j}}, 0,0\right)=0$, then $u_{\mathcal{T}_{j}}=0$ and $\psi_{\mathcal{T}_{j}}=0$. We multiply equation (2.7a) by $m_{\mathrm{K}} u_{\mathrm{K}}$ and equations $(2.7 \mathrm{~b})-(2.7 \mathrm{~d})$ by $m_{\mathrm{K}^{*}} u_{\mathrm{K}^{*}}$ and sum these identities over all the control volumes in $\mathfrak{M}_{j}$ and $\mathfrak{M}_{j}^{*} \cup \partial \mathfrak{M}_{j, \Gamma}^{*}$. Reordering the different contributions over all diamond cells, we obtain

$$
\begin{aligned}
& 2 \sum_{\mathrm{D} \in \mathfrak{D}_{j}} m_{\mathrm{D}}\left(A_{\mathrm{D}} \nabla^{\mathrm{D}} u_{\mathcal{T}_{j}}, \nabla^{\mathrm{D}} u_{\mathcal{T}_{j}}\right)+p\left(u_{\mathcal{T}_{j}}, u_{\mathcal{T}_{j}}\right)_{\partial \mathfrak{M}_{j, \Gamma}} \\
& \quad+p^{*}\left(u_{\mathcal{T}_{j}}, u_{\mathcal{T}_{j}}\right){ }_{\partial \mathfrak{M}_{j, \Gamma}^{*}}+\sum_{\mathrm{K} \in \mathfrak{M}_{j}} m_{\mathrm{K}} \eta_{\mathrm{K}} u_{\mathrm{K}}^{2}+\sum_{\mathrm{K}^{*} \in \mathfrak{M}_{j}^{*} \cup \partial \mathfrak{M}_{j, \Gamma}^{*}} m_{\mathrm{K}^{*}} \eta_{\mathrm{K}^{*}} u_{\mathrm{K}^{*}}^{2}=0,
\end{aligned}
$$

where

$$
\begin{aligned}
\left(u_{\mathcal{T}_{j}}, v_{\mathcal{T}_{j}}\right)_{\partial \mathfrak{M}_{j, \Gamma}} & :=\sum_{\mathrm{L} \in \partial \mathfrak{M}_{j, \Gamma}} m_{\sigma} u_{\mathrm{L}} v_{\mathrm{L}}=\sum_{i \in I_{j} \mathrm{~L} \in \partial \mathfrak{M}_{j, \Gamma_{i}}} m_{\sigma} u_{\mathrm{L}} v_{\mathrm{L}}, \\
\left(u_{\mathcal{T}_{j}}, v_{\mathcal{T}_{j}}\right)_{\partial \mathfrak{M}_{j, \Gamma}^{*}} & :=\sum_{i \in I_{j} \mathrm{~K}^{*} \in \partial \mathfrak{M}_{j, \Gamma_{i}}^{*}} m_{\sigma_{\mathrm{K}^{*}}} u_{\mathrm{K}^{*}} v_{\mathrm{K}^{*}}+\sum_{\mathrm{K}^{*}=\mathrm{K}_{i k}^{*} \in \partial \mathfrak{M}_{j, C}^{*}}\left(m_{\sigma_{j, \mathrm{~K}^{*}}^{i}}+m_{\sigma_{j, \mathrm{~K}^{*}}^{k}}\right) u_{\mathrm{K}^{*}} v_{\mathrm{K}^{*}} .
\end{aligned}
$$

Since all the terms are positive, we obtain if $\eta>0$ from the last two terms in (2.8) that $u_{\mathcal{T}_{j}}=0$. If $\eta=0$, we get first from (2.8) that $u_{\mathcal{\tau}_{j}}$ vanishes on the boundary $\partial \mathfrak{M}_{j, \Gamma} \cup \partial \mathfrak{M}_{j, \Gamma}^{*}$, since $p>0$ and $p^{*}>0$. Furthermore, since $A$ is coercive, (2.8) also shows that $\nabla^{\mathrm{D}} u_{\mathcal{T}_{j}}$ vanishes, and thus $u_{\mathcal{T}_{j}}=0$ because a DDFV Discrete Poincaré inequality proved in [1] gives

$$
\sum_{\mathrm{K} \in \mathfrak{M}_{j}} m_{\mathrm{K}} u_{\mathrm{K}}^{2}+\sum_{\mathrm{K}^{*} \in \mathfrak{M}_{j}^{*} \cup \partial \mathfrak{M}_{j, \Gamma}^{*}} m_{\mathrm{K}^{*}} u_{\mathrm{K}^{*}}^{2} \leq C \sum_{\mathrm{D} \in \mathfrak{D}} m_{\mathrm{D}}\left|\nabla^{\mathrm{D}} u_{\mathcal{T}_{j}}\right|^{2}
$$

as soon as $u_{\mathcal{T}_{j}}$ vanishes on part of $\partial \mathfrak{M}_{j}$ and part of $\partial \mathfrak{M}_{j}^{*}$. We finally obtain $\psi_{\mathcal{T}_{j}}=0$ using the transmission conditions $(2.7 \mathrm{f})$ and $(2.7 \mathrm{~g})$, which are homogeneous. 


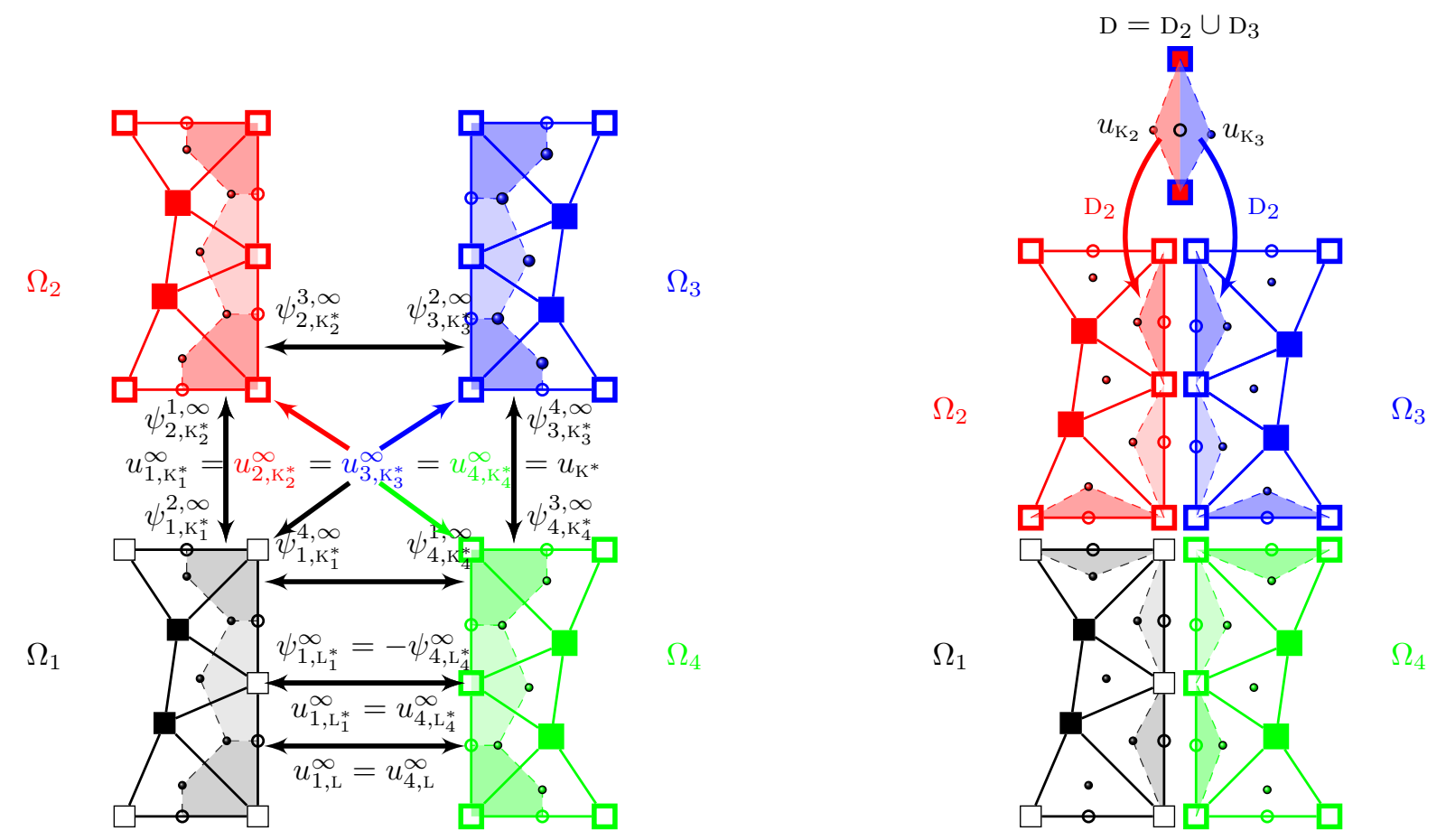

Figure 3: Left: new unknowns needed to describe the DDFV scheme on $\Omega$ as the limit of the Schwarz algorithm. Right: splitting of the diamond cells at the interfaces.

\subsection{DDFV Schwarz Algorithm for Anisotropic Diffusion}

We can now present the optimized Schwarz algorithm discretized by DDFV: for $f_{\mathcal{T}_{j}} \in \mathbb{R}^{\mathfrak{M}_{j} \cup \mathfrak{M}_{j}^{*} \cup \partial \mathfrak{M}_{j, \Gamma}^{*}}$

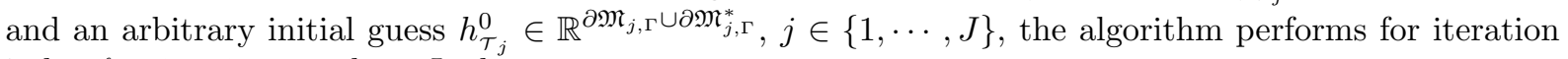
index $\ell=0,1,2, \ldots$ and $i \in I_{j}$ the two steps:

1. Compute the subdomain solutions $\left(u_{\mathcal{T}_{j}}^{\ell+1}, \psi_{\mathcal{T}_{j}}^{\ell+1}\right) \in \mathbb{R}^{\mathcal{T}_{j}} \times \mathbb{R}^{\partial \mathfrak{M}_{j, \Gamma}^{*}}$ by solving for $j=1,2, \ldots, J$

$$
\mathcal{L}^{\mathcal{T}_{j}}\left(u_{\mathcal{T}_{j}}^{\ell+1}, \psi_{\mathcal{T}_{j}}^{\ell+1}, f_{\mathcal{T}_{j}}, h_{\mathcal{T}_{j}}^{\ell}\right)=0
$$

2. Compute the new values $h_{\mathcal{T}_{j}}^{\ell+1}$ to be transmitted to neighboring subdomains,

$$
\begin{aligned}
h_{j, \mathrm{~L}}^{\ell+1} & =-\frac{1}{m_{\sigma}}\left(A_{\mathrm{D}} \nabla^{\mathrm{D}} u_{\mathcal{T}_{i}}^{\ell+1}, \mathbf{N}_{\mathrm{KL}}\right)+p u_{i, \mathrm{~L}}^{\ell+1}, \forall \mathrm{L} \in \partial \mathfrak{M}_{j, \Gamma_{i}}, \forall i \in I_{j}, \\
h_{j, \mathrm{~K}^{*}}^{\ell+1} & =-\psi_{i, \mathrm{~L}^{*}}^{\ell+1}+p^{*} u_{i, \mathrm{~L}^{*}}^{\ell+1}, \forall \mathrm{K}^{*} \in \partial \mathfrak{M}_{j, \Gamma_{i}, i n t}^{*}, \forall \mathrm{L}^{*} \in \partial \mathfrak{M}_{i, \Gamma_{j}, i n t}^{*} \text { s.t. } x_{\mathrm{K}^{*}}=x_{\mathrm{L}^{*}}, \forall i \in I_{j}, \\
h_{j, \mathrm{~K}^{*}}^{i,+1} & =-\psi_{i, \mathrm{~L}^{*}}^{j+1}+p^{*} u_{i, \mathrm{~L}^{*}}^{\ell+1}, \forall \mathrm{K}^{*} \in \partial \mathfrak{M}_{j, C}^{*}, \forall \mathrm{L}^{*} \in \partial \mathfrak{M}_{i, C}^{*} \text { s.t. } x_{\mathrm{K}^{*}}=x_{\mathrm{L}^{*}}, \forall i \in I_{j} .
\end{aligned}
$$

We can now prove convergence of the non-overlapping DDFV optimized Schwarz algorithm with Robin transmission conditions. We denote by $\mathcal{T}=\left(\mathfrak{M}, \partial \mathfrak{M}, \mathfrak{M}^{*}, \partial \mathfrak{M}^{*}\right)$ the DDFV mesh constructed from the primal discretization of the sub-domain $\Omega_{j}: \mathfrak{M}=\cup \mathfrak{M}_{j}$.

Theorem 2.2 (Convergence of the DDFV Schwarz algorithm). The iterates of the optimized Schwarz algorithm discretized by DDFV defined by (2.11)-(2.12) converge as $\ell$ tends to infinity to the solution $u_{\mathcal{T}}$ of the DDFV scheme (2.5) on $\Omega$.

Proof. The crucial step of the proof consists in rewriting the classical DDFV scheme (2.5) on $\Omega$ as the limit of the Schwarz algorithm. To this end, we introduce new unknowns near the interface $\Gamma_{j i}$, see Figure 3: 
- for all $\mathrm{K} \in \mathfrak{M}_{j}$ and $\mathrm{K}^{*} \in \mathfrak{M}_{j}^{*}$, we set $u_{\mathrm{K}}^{\infty}:=u_{\mathrm{K}}$ and $u_{\mathrm{K}^{*}}^{\infty}:=u_{\mathrm{K}^{*}}$,

- for all $\mathrm{K} \in \partial \mathfrak{M}_{j, D}$ and $\mathrm{K}^{*} \in \partial \mathfrak{M}_{j, D}^{*}$, we set $u_{\mathrm{K}}^{\infty}:=0$ and $u_{\mathrm{K}^{*}}^{\infty}:=0$,

- for all $\mathrm{L}=\mathrm{K}_{j} \mid \mathrm{K}_{i} \in \partial \mathfrak{M}_{j, \Gamma_{i}}=\partial \mathfrak{M}_{i, \Gamma_{j}}$, define

$$
u_{j, \mathrm{~L}}^{\infty}=u_{i, \mathrm{~L}}^{\infty}:=\frac{m_{\mathrm{D}_{i}} u_{\mathrm{K}_{j}}+m_{\mathrm{D}_{j}} u_{\mathrm{K}_{i}}}{m_{\mathrm{D}}},
$$

so that $\left(A_{\mathrm{D}_{j}} \nabla^{\mathrm{D}_{j}} u_{\mathcal{T}_{j}}^{\infty}, \mathbf{N}_{\mathrm{K}_{j} \mathrm{~L}}\right)=-\left(A_{\mathrm{D}_{i}} \nabla^{\mathrm{D}_{i}} u_{\mathcal{T}_{i}}^{\infty}, \mathbf{N}_{\mathrm{K}_{i} \mathrm{~L}}\right)$, with $\mathrm{D}_{j} \in \mathfrak{D}_{j, \Gamma}$ and $\mathrm{D}_{i} \in \mathfrak{D}_{i, \Gamma}$, with $A_{\mathrm{D}_{j}}=$ $A_{\mathrm{D}_{i}}=A_{\mathrm{D}}$ as $x_{\mathrm{D}_{j}}=x_{\mathrm{D}_{i}}=x_{\mathrm{L}}$.

- for all $\mathrm{K}^{*} \in \mathfrak{M}^{*}$ such that $x_{\mathrm{K}^{*}} \in \Gamma_{i j}$, i.e. $\mathrm{K}^{*}=\mathrm{K}_{i}^{*} \cup \mathrm{K}_{j}^{*}$ with $\mathrm{K}_{j}^{*} \in \partial \mathfrak{M}_{j, \Gamma_{i}, \text { int }}^{*}$ and $\mathrm{K}_{i}^{*} \in \partial \mathfrak{M}_{i, \Gamma_{j}, \text { int }}^{*}$, set $u_{j, \mathrm{~K}_{j}^{*}}^{\infty}=u_{i, \mathrm{~K}_{i}^{*}}^{\infty}:=u_{\mathrm{K}^{*}}$ and

$$
\begin{aligned}
\psi_{j, \mathrm{~K}_{j}^{*}}^{\infty}=-\psi_{i, \mathrm{~K}_{i}^{*}}^{\infty}:=-\frac{1}{m_{\sigma_{\mathrm{K}^{*}}}} \sum_{\mathrm{D} \in \mathfrak{D}_{\mathrm{K}_{j}^{*}}}\left(A_{\mathrm{D}} \nabla^{\mathrm{D}} u_{\mathcal{T}_{j}}^{\infty}, \mathbf{N}_{\mathrm{K}_{j}^{*} L_{j}^{*}}\right)+\frac{m_{\mathrm{K}_{j}^{*}}}{m_{\sigma_{\mathrm{K}^{*}}}}\left(\eta_{\mathrm{K}^{*}} u_{\mathrm{K}^{*}}-f_{\mathrm{K}^{*}}\right) \\
=\frac{1}{m_{\sigma_{\mathrm{K}^{*}}}} \sum_{\mathrm{D} \in \mathfrak{D}_{\mathrm{K}_{i}^{*}}}\left(A_{\mathrm{D}} \nabla^{\mathrm{D}} u_{\mathcal{T}_{i}}^{\infty}, \mathbf{N}_{\mathrm{K}_{i}^{*} \mathrm{~L}_{i}^{*}}\right)-\frac{m_{\mathrm{K}_{i}^{*}}}{m_{\sigma_{\mathrm{K}^{*}}}}\left(\eta_{\mathrm{K}^{*}} u_{\mathrm{K}^{*}}-f_{\mathrm{K}^{*}}\right) .
\end{aligned}
$$

Equation (2.13) comes from equation (2.5b), in which we have split the terms from $\mathrm{K}_{i}^{*}$ and $\mathrm{K}_{j}^{*}$, noting that $m_{\mathrm{K}^{*}}=m_{\mathrm{K}_{j}^{*}}+m_{\mathrm{K}_{i}^{*}}$.

- At a cross point $c$, we denote by $I_{c}$ the set of indices of the subdomains that intersect in that cross point. The cross point is the center of a dual cell $\mathrm{K}^{*}$ that is split in the domain decomposition. We define for each subcell $\mathrm{K}_{j}^{*} \subset \Omega_{j}, j \in I_{c}$ an unknown $u_{j, \mathrm{~K}_{j}^{*}}^{\infty}:=u_{\mathrm{K}^{*}}$. We also have to introduce for all $j \in I_{c}$ additional unknowns $\psi_{j, \mathrm{~K}_{j}^{*}}^{i, \infty}$ and $\psi_{j, \mathrm{~K}_{j}^{*}}^{k, \infty}$ in such a way that

$$
m_{\sigma_{j, \mathrm{~K}_{j}^{*}}^{i}} \psi_{j, \mathrm{~K}_{j}^{*}}^{i, \infty}+m_{\sigma_{j, \mathrm{~K}_{j}^{*}}^{k}} \psi_{j, \mathrm{~K}_{j}^{*}}^{k, \infty}=\sum_{\mathrm{D} \in \mathfrak{D}_{\mathrm{K}_{j}^{*}}}\left(A_{\mathrm{D}} \nabla^{\mathrm{D}} u_{\mathcal{T}_{j}}, \mathbf{N}_{\mathrm{K}^{*} \mathrm{~L}^{*}}\right)-m_{\mathrm{K}_{j}^{*}} \eta_{\mathrm{K}^{*}} u_{j, \mathrm{~K}^{*}}+m_{\mathrm{K}_{j}^{*}} f_{\mathrm{K}^{*}}=: b_{j} .
$$

According to the DDFV scheme $(2.5), \sum_{j \in I_{c}} b_{j}=0$. We denote by $n_{c}$ the cardinal of $I_{c}$. Now imposing $\psi_{j, \mathrm{~K}_{j}^{*}}^{i, \infty}=-\psi_{i, \mathrm{~K}_{i}^{*}}^{j, \infty}$, these fluxes must satisfy a linear system of the form $B \psi=b$ with $B$ an $n_{c} \times n_{c}$ matrix, $b=\left(b_{j}\right)_{j \in I_{c}}$ and $\psi$ the vector of $\psi_{j, \mathrm{~K}_{j}^{*}}^{i, \infty}$ after selection of $n_{c}$ out of the $2 n_{c}$ possible ones, see [20] where this technique to treat cross points was introduced. For example, in the case $n_{c}=4, I_{c}=\{1,2,3,4\}$ with $\Omega_{j}$ arranged clockwise, we can take

$$
\psi=\left(\begin{array}{c}
\psi_{1, \mathrm{~K}_{1}^{*}}^{2, \infty} \\
\psi_{2, \mathrm{~K}_{2}^{*}}^{3, \infty} \\
\psi_{3, \infty}^{4, \infty} \\
\psi_{4, \mathrm{~K}_{4}^{*}}^{1, \infty}
\end{array}\right), \quad B=\left(\begin{array}{cccc}
m_{\sigma_{1, \mathrm{~K}_{1}^{*}}^{2}} & 0 & 0 & -m_{\sigma_{4, \mathrm{~K}_{4}^{*}}^{1}} \\
-m_{\sigma_{1, \mathrm{~K}_{1}^{*}}^{2}} & m_{\sigma_{2, \mathrm{~K}_{2}^{*}}^{3}} & 0 & 0 \\
0 & -m_{\sigma_{2, \mathrm{~K}}^{*}}^{3} & m_{\sigma_{3, \mathrm{~K}_{3}^{*}}^{4}} & 0 \\
0 & 0 & -m_{\sigma_{3, \mathrm{~K}_{3}^{*}}^{4}} & m_{\sigma_{4, \mathrm{~K}_{4}^{*}}^{1}}
\end{array}\right) .
$$

The rank of $B$ is equal to $n_{c}-1$ and $b \in \operatorname{Im} B$ since $\sum_{j \in I_{c}} b_{j}=0$. Therefore, $\psi$ exists but is not unique. The $\psi$ at the cross point will thus in general not converge in the optimized Schwarz algorithm, which does however not affect the convergence of the $u$, see [20].

- Therefore, we can define

$$
\begin{aligned}
h_{j, \mathrm{~L}}^{\infty} & =-\frac{1}{m_{\sigma}}\left(A_{\mathrm{D}} \nabla^{\mathrm{D}} u_{\mathcal{T}_{i}}^{\infty}, \mathbf{N}_{\mathrm{KL}}\right)+p u_{i, \mathrm{~L}}^{\infty}, \forall \mathrm{L} \in \partial \mathfrak{M}_{j, \Gamma_{i}}, \forall i \in I_{j}, \\
h_{j, \mathrm{~K}^{*}}^{\infty} & =-\psi_{i, \mathrm{~L}^{*}}^{\infty}+p^{*} u_{i, \mathrm{~L}^{*}}^{\infty}, \forall \mathrm{K}^{*} \in \partial \mathfrak{M}_{j, \Gamma_{i}, i n t}^{*}, \forall \mathrm{L}^{*} \in \partial \mathfrak{M}_{i, \Gamma_{j}, i n t}^{*} \text { s.t. } x_{\mathrm{K}^{*}}=x_{\mathrm{L}^{*}}, \forall i \in I_{j}, \\
h_{j, \mathrm{~K}^{*}}^{i, \infty} & =-\psi_{i, \mathrm{~L}^{*}}^{j, \infty}+p^{*} u_{i, \mathrm{~L}^{*}}^{\infty}, \forall \mathrm{K}^{*} \in \partial \mathfrak{M}_{j, C}^{*}, \forall \mathrm{L}^{*} \in \partial \mathfrak{M}_{i, C}^{*} \text { s.t. } x_{\mathrm{K}^{*}}=x_{\mathrm{L}^{*}}, \forall i \in I_{j} .
\end{aligned}
$$


We have constructed $\left(u_{\mathcal{T}_{j}}^{\infty}, \psi_{\mathcal{T}_{j}}^{\infty}\right)$ from the solution $u_{\mathcal{T}}$ of the DDFV scheme (2.5) on $\Omega$ such that

$$
\mathcal{L}^{\mathcal{T}_{j}}\left(u_{\mathcal{T}_{j}}^{\infty}, \psi_{\mathcal{T}_{j}}^{\infty}, f_{\mathcal{T}_{j}}, h_{\mathcal{T}_{j}}^{\infty}\right)=0 .
$$

Observe that the errors $e_{\mathcal{T}_{j}}^{\ell+1}:=u_{\mathcal{T}_{j}}^{\ell+1}-u_{\mathcal{T}_{j}}^{\infty}, \Psi_{\mathcal{T}_{j}}^{\ell+1}:=\psi_{\mathcal{T}_{j}}^{\ell+1}-\psi_{\mathcal{T}_{j}}^{\infty}$ satisfy

$$
\mathcal{L}^{\mathcal{T}_{j}}\left(e_{\mathcal{T}_{j}}^{\ell+1}, \Psi_{\mathcal{T}_{j}}^{\ell+1}, 0, H_{\mathcal{T}_{j}}^{\ell}\right)=0
$$

with

$$
\begin{aligned}
H_{j, \mathrm{~L}}^{\ell} & =-\frac{1}{m_{\sigma}}\left(A_{\mathrm{D}} \nabla^{\mathrm{D}} e_{\mathcal{T}_{i}}^{\ell}, \mathbf{N}_{\mathrm{KL}}\right)+p e_{i, \mathrm{~L}}^{\ell}, \forall \mathrm{L} \in \partial \mathfrak{M}_{j, \Gamma_{i}}, \forall i \in I_{j}, \\
H_{j, \mathrm{~K}^{*}}^{\ell} & =-\Psi_{i, \mathrm{~L}^{*}}^{\ell}+p^{*} e_{i, \mathrm{~L}^{*}}^{\ell}, \forall \mathrm{K}^{*} \in \partial \mathfrak{M}_{j, \Gamma_{i}, i n t}^{*}, \forall \mathrm{L}^{*} \in \partial \mathfrak{M}_{i, \Gamma_{j}, i n t}^{*} \text { s.t. } x_{\mathrm{K}^{*}}=x_{\mathrm{L}^{*}}, \forall i \in I_{j}, \\
H_{j, \mathrm{~K}^{*}}^{i, \ell} & =-\Psi_{i, \mathrm{~L}^{*}}^{j, \ell}+p^{*} e_{i, \mathrm{~L}^{*}}^{\ell}, \forall \mathrm{K}^{*} \in \partial \mathfrak{M}_{j, C}^{*}, \forall \mathrm{L}^{*} \in \partial \mathfrak{M}_{i, C}^{*} \text { s.t. } x_{\mathrm{K}^{*}}=x_{\mathrm{L}^{*}}, \forall i \in I_{j} .
\end{aligned}
$$

For $j=1, \cdots, J$, we multiply equation (2.7a) associated to the scheme (2.16) by $m_{\mathrm{K}} e_{\mathrm{K}}$ and equations (2.7b)-(2.7d) by $m_{\mathrm{K}^{*}} e_{\mathrm{K}^{*}}$ and sum these identities over all the control volumes in $\mathfrak{M}_{j}$ and $\mathfrak{M}_{j}^{*} \cup \partial \mathfrak{M}_{j, \Gamma}^{*}$. Reordering the different contributions over all diamond cells, we obtain

$$
\begin{aligned}
& 2 \sum_{\mathrm{D} \in \mathfrak{D}_{j}} m_{\mathrm{D}}\left(A_{\mathrm{D}} \nabla^{\mathrm{D}} e_{\mathcal{T}_{j}}^{\ell+1}, \nabla^{\mathrm{D}} e_{\mathcal{T}_{j}}^{\ell+1}\right)+\sum_{\mathrm{K} \in \mathfrak{M}_{j}} m_{\mathrm{K}} \eta_{\mathrm{K}}\left(e_{j, \mathrm{~K}}^{\ell+1}\right)^{2}+\sum_{\mathrm{K}^{*} \in \mathfrak{M}_{j}^{*} \cup \partial \mathfrak{M}_{j, \Gamma}^{*}} m_{\mathrm{K}^{*}} \eta_{\mathrm{K}^{*}}\left(e_{j, \mathrm{~K}^{*}}^{\ell+1}\right)^{2} \\
& \quad-\sum_{\mathrm{L} \in \partial \mathfrak{M}_{j, \Gamma}}\left(A_{\mathrm{D}} \nabla^{\mathrm{D}} e_{\mathcal{T}_{j}}^{\ell+1}, \mathbf{N}_{\mathrm{KL}}\right) e_{j, \mathrm{~L}}^{\ell+1}-\sum_{\mathrm{K}^{*} \in \partial \mathfrak{M}_{j, \Gamma, i n t}^{*}} m_{\sigma_{\mathrm{K}^{*}}} \Psi_{\mathrm{K}^{*}}^{\ell+1} e_{j, \mathrm{~K}^{*}}^{\ell+1} \\
& \quad-\sum_{\mathrm{K}^{*}=\mathrm{K}_{i k}^{*} \in \partial \mathfrak{M}_{j, C}^{*}}\left(m_{\sigma_{j, \mathrm{~K}}^{*}}^{k} \Psi_{j, \mathrm{~K}^{*}}^{k, \ell+1}+m_{\sigma_{j, \mathrm{~K}^{*}}^{i}} \Psi_{j, \mathrm{~K}^{*}}^{i+1}\right) e_{j, \mathrm{~K}^{*}}^{\ell+1}=0 .
\end{aligned}
$$

Now we apply the identity $-a b=\frac{1}{4}\left((a-b)^{2}-(a+b)^{2}\right)$, used for such estimates in $[27,8]$, to the four terms

$$
\left(A_{\mathrm{D}} \nabla^{\mathrm{D}} e_{\mathcal{T}_{j}}^{\ell+1}, \mathbf{N}_{\mathrm{KL}}\right) e_{j, \mathrm{~L}}^{\ell+1}, \quad m_{\sigma_{\mathrm{K}^{*}}} \Psi_{\mathrm{K}^{*}}^{\ell+1} e_{j, \mathrm{~K}^{*}}^{\ell+1}, \quad m_{\sigma_{j, \mathrm{~K}^{*}}^{k}} \Psi_{j, \mathrm{~K}^{*}}^{k, \ell+1} e_{j, \mathrm{~K}^{*}}^{\ell+1}, \quad m_{\sigma_{j, \mathrm{~K}^{*}}^{i}} \Psi_{j, \mathrm{~K}^{*}}^{i, \ell+1} e_{j, \mathrm{~K}^{*}}^{\ell+1},
$$

the transmission conditions appear, for example

$$
m_{\sigma_{j, \mathrm{~K}^{*}}^{i}} \Psi_{j, \mathrm{~K}^{*}}^{i, \ell+1} e_{j, \mathrm{~K}^{*}}^{\ell+1}=\frac{m_{\sigma_{j, \mathrm{~K}^{*}}^{i}}}{4 p^{*}}\left(\left(\Psi_{j, \mathrm{~K}^{*}}^{i, \ell+1}+p^{*} e_{j, \mathrm{~K}^{*}}^{\ell+1}\right)^{2}-\left(-\Psi_{j, \mathrm{~K}^{*}}^{i, \ell+1}+p^{*} e_{j, \mathrm{~K}^{*}}^{\ell+1}\right)^{2}\right),
$$

and thus using $(2.17 \mathrm{c})$, we get

$$
m_{\sigma_{j, \mathrm{~K}^{*}}^{i}} \Psi_{j, \mathrm{~K}^{*}}^{i, \ell+1} e_{j, \mathrm{~K}^{*}}^{\ell+1}=\frac{m_{\sigma_{j, \mathrm{~K}^{*}}^{i}}}{4 p^{*}}\left(\left(-\Psi_{i, \mathrm{~K}^{*}}^{j, \ell}+p^{*} e_{i, \mathrm{~K}^{*}}^{\ell}\right)^{2}-\left(-\Psi_{j, \mathrm{~K}^{*}}^{i, \ell+1}+p^{*} e_{j, \mathrm{~K}^{*}}^{\ell+1}\right)^{2}\right) .
$$

Summing now over all iteration indices $\ell=0, \ldots, \ell_{\max }-1$ and subdomain indices $j=1, \ldots, J$ we get for all $\mathrm{K}^{*}=\mathrm{K}_{i k}^{*} \in \partial \mathfrak{M}_{j, C}^{*}$ using $m_{\sigma_{j, \mathrm{~K}^{*}}^{i}}=m_{\sigma_{i, \mathrm{~K}^{*}}^{j}}$

$$
\begin{aligned}
-\sum_{\ell=0}^{\ell_{\max }-1} \sum_{j=1}^{J} m_{\sigma_{j, \mathrm{~K}^{*}}} \Psi_{j, \mathrm{~K}^{*}}^{i, \ell+1} e_{j, \mathrm{~K}^{*}}^{\ell+1} & =\frac{1}{4 p^{*}} \sum_{\ell=0}^{\ell_{\max }-1} \sum_{j=1}^{J} m_{\sigma_{j, \mathrm{~K}^{*}}^{i}}\left(-\left(-\Psi_{i, \mathrm{~K}^{*}}^{j, \ell}+p^{*} e_{i, \mathrm{~K}^{*}}^{\ell}\right)^{2}+\left(-\Psi_{j, \mathrm{~K}^{*}}^{i, \ell+1}+p^{*} e_{j, \mathrm{~K}^{*}}^{\ell+1}\right)^{2}\right) \\
& =\frac{1}{4 p^{*}} \sum_{j=1}^{J} m_{\sigma_{j, \mathrm{~K}^{*}}^{i}}\left(-\left(-\Psi_{j, \mathrm{~K}^{*}}^{j, 0}+p^{*} e_{j, \mathrm{~K}^{*}}^{0}\right)^{2}+\left(-\Psi_{j, \mathrm{~K}^{*}}^{i, \ell_{\max }}+p^{*} e_{j, \mathrm{~K}^{*}}^{\ell_{\max }}\right)^{2}\right) .
\end{aligned}
$$

Similarly,

$$
-\sum_{\ell=0}^{\ell_{\max }-1} \sum_{j=1}^{J} m_{\sigma_{j, \mathrm{~K}^{*}}^{k}} \Psi_{j, \mathrm{~K}^{*}}^{k, \ell+1} e_{j, \mathrm{~K}^{*}}^{\ell+1}=\frac{1}{4 p^{*}} \sum_{j=1}^{J} m_{\sigma_{j, \mathrm{~K}^{*}}^{k}}\left(-\left(-\Psi_{j, \mathrm{~K}^{*}}^{j, 0}+p^{*} e_{j, \mathrm{~K}^{*}}^{0}\right)^{2}+\left(-\Psi_{j, \mathrm{~K}^{*}}^{k, \ell_{\max }}+p^{*} e_{j, \mathrm{~K}^{*}}^{\ell_{\max }}\right)^{2}\right) .
$$


For all $\mathrm{K}^{*} \in \partial \mathfrak{M}_{j, \Gamma, i n t}^{*}$, we have

$$
-\sum_{\ell=0}^{\ell_{\max }-1} \sum_{j=1}^{J} m_{\sigma_{\mathrm{K}^{*}}} \Psi_{\mathrm{K}^{*}}^{\ell+1} e_{j, \mathrm{~K}^{*}}^{\ell+1}=\frac{1}{4 p^{*}} \sum_{j=1}^{J} m_{\sigma_{\mathrm{K}^{*}}}\left(-\left(-\Psi_{j, \mathrm{~K}^{*}}^{j, 0}+p^{*} e_{j, \mathrm{~K}^{*}}^{0}\right)^{2}+\left(-\Psi_{j, \mathrm{~K}^{*}}^{\ell_{\max }}+p^{*} e_{j, \mathrm{~K}^{*}}^{\ell_{\max }}\right)^{2}\right),
$$

and for all $\mathrm{L} \in \partial \mathfrak{M}_{j, \Gamma}$, we have

$$
-\sum_{\ell=0}^{\ell_{\max }-1} \sum_{j=1}^{J}\left(A_{\mathrm{D}} \nabla^{\mathrm{D}} e_{\mathcal{T}_{j}}^{\ell+1}, \mathbf{N}_{\mathrm{KL}}\right) e_{j, \mathrm{~L}}^{\ell+1}=\frac{1}{4 p^{*}} \sum_{j=1}^{J}\left(-\left(-\left(A_{\mathrm{D}} \nabla^{\mathrm{D}} e_{\mathcal{T}_{j}}^{0}, \mathbf{N}_{\mathrm{KL}}\right)+p e_{j, \mathrm{~L}}^{0}\right)^{2}+\left(-\left(A_{\mathrm{D}} \nabla^{\mathrm{D}} e_{\mathcal{T}_{j}}^{\ell_{\max }}, \mathbf{N}_{\mathrm{KL}}\right)+p e_{j, \mathrm{~L}}^{\ell_{\max }}\right)^{2}\right) .
$$

Gathering theses contributions, we obtain

$$
\begin{aligned}
& 2 \sum_{\ell=0}^{\ell_{\max }-1} \sum_{j=1}^{J} \sum_{\mathfrak{D} \in \mathfrak{D}_{j}} m_{\mathrm{D}}\left(A_{\mathrm{D}} \nabla^{\mathrm{D}} e_{\mathcal{T}_{j}}^{\ell+1}, \nabla^{\mathrm{D}} e_{\mathcal{T}_{j}}^{\ell+1}\right)+\sum_{\ell=0}^{\ell_{\max }-1} \sum_{j=1}^{J} \sum_{\mathrm{K} \in \mathfrak{M}_{j}} m_{\mathrm{K}} \eta_{\mathrm{K}}\left(e_{\mathrm{K}}^{\ell+1}\right)^{2}+\sum_{\ell=0}^{\ell_{\max }-1} \sum_{j=1}^{J} \sum_{\mathrm{K}^{*} \in \mathfrak{M}_{j}^{*} \cup \partial \mathfrak{M}_{j, \Gamma}^{*}} m_{\mathrm{K}^{*}} \eta_{\mathrm{K}^{*}}\left(e_{\mathrm{K}^{*}}^{\ell+1}\right)^{2} \\
& \quad+\frac{1}{4 p} \sum_{j=1}^{J}\left\|-\left(A_{\mathfrak{D}_{j, \Gamma}} \nabla^{\mathfrak{D}_{j, \Gamma}} e_{\mathcal{T}_{j}}^{\ell_{\max }}, \mathbf{n}_{j}\right)+p e_{\partial \mathfrak{M}_{j, \Gamma} \ell_{\max }}\right\|_{\partial \mathfrak{M}_{j, \Gamma}}^{2}+\frac{1}{4 p^{*}} \sum_{j=1}^{J}\left\|-\Psi_{\mathcal{T}_{j}}^{\ell_{\max }}+p^{*} e_{\partial \mathfrak{M}_{j, \Gamma}^{*}}^{\ell_{\max }}\right\|_{\partial \mathfrak{M}_{j, \Gamma}^{*}}^{2} \\
& \quad \leq \frac{1}{4 p} \sum_{j=1}^{J}\left\|-\left(A_{\mathfrak{D}_{j, \Gamma}} \nabla^{\mathfrak{D}_{j, \Gamma}} e_{\mathcal{T}_{j}}^{0}, \mathbf{n}_{j}\right)+p e_{\partial \mathfrak{M}_{j, \Gamma}}^{0}\right\|_{\partial \mathfrak{M}_{j, \Gamma}}^{2}+\frac{1}{4 p^{*}} \sum_{j=1}^{J}\left\|-\Psi_{\mathcal{T}_{j}}^{0}+p^{*} e_{\partial \mathfrak{M}_{j, \Gamma}^{*}}^{0}\right\|_{\partial \mathfrak{M}_{j, \Gamma}^{*}}^{2},
\end{aligned}
$$

where $\|\cdot\|_{\partial \mathfrak{M}_{j, \Gamma}}$ and $\|\cdot\|_{\partial \mathfrak{M}_{j, \Gamma}^{*}}$ are the norms associated to the scalar products defined in (2.9) and (2.10). This shows that the total discrete energy stays bounded as the iteration index $\ell$ goes to infinity, and hence the discrete $H^{1}$ norm of $e_{\mathcal{T}_{j}}^{\ell+1}$ converges to zero as $\ell$ tends to infinity for all $j$. In other words, the iterates $u_{\mathcal{T}_{j}}^{\ell}$ of the optimized Schwarz algorithm discretized by DDFV defined by (2.11)-(2.12) converge as $\ell$ tends to infinity to $u_{\mathcal{T}_{j}}^{\infty}$. Coming back to the construction of $u_{\mathcal{T}_{j}}^{\infty}$, we obtain the convergence to $u_{\mathcal{T}}$ the solution of the DDFV scheme (2.5) on $\Omega$. Note that the estimate only gives a bound on the discrete fluxes $\Psi_{\mathcal{T}_{j}}^{\ell}$ and in practice the discrete fluxes do not converge at cross points, because the flux system there is not full rank, see (2.14).

\section{Optimization of the Robin Transmission Conditions}

We now present for the first time a discrete optimization of the Robin parameter in the transmission conditions of the DDFV Schwarz algorithm (2.11), (2.15a)-(2.15c) in order to understand why the optimized parameters from the continuous analysis in [17] sometimes give suboptimal performance for high anisotropies. As in the continuous case, we focus on a two subdomain decomposition, and will use the same parameters also at cross points. There are also cross point formulations where this is not advised, see [19].

\subsection{Discrete Subdomain Solutions}

To obtain a discrete convergence factor, we use the typical approach in optimized Schwarz methods to consider a domain $\Omega:=(-a, a) \times(0, b)$ decomposed into two non-overlapping subdomains $\Omega_{1}:=$ $(-a, 0) \times(0, b)$ and $\Omega_{2}:=(0, a) \times(0, b)$, with the interface $\Gamma:=\partial \Omega_{1} \cap \partial \Omega_{2}$. We use a rectangular grid, so that the DDFV discretization away from the interface $\Gamma$ leads to two interlaced five point finite difference schemes. The mesh size is denoted by $\left(h_{x}, h_{y}\right)$. To simplify the notation compared to the general DDFV scheme, we use for the dual (vertex centered) unknowns aligned with the interface star indices, $u_{m^{*}, n^{*}}^{j, \ell}$. These are associated with the dual cells shown in dashed Figure 4, whose centers are squares ( $\mathbf{\square}$ or $\square$ ), and the superscripts $j$ and $\ell$ stand for the domain and the iteration. For the primal (cell centered) unknowns, we use indices without stars, $u_{m, n}^{j, \ell}$. These are associated with the primal cells, whose centers are bullets $\left(\bullet\right.$ or $\circ$ for interface cells), see Figure 4. Additional primal unknowns $u_{\frac{1}{2}, n}^{j, \ell}$, located at $\circ$ in Figure 4 , and also additional flux unknowns $\psi_{n^{*}}^{j, \ell}$ are needed on the interface $\Gamma$. We study directly the error $u_{m, n}-u_{m, n}^{j, \ell}$ which satisfies the same algorithm as $u_{m, n}^{j, \ell}$ but with zero source term and boundary 


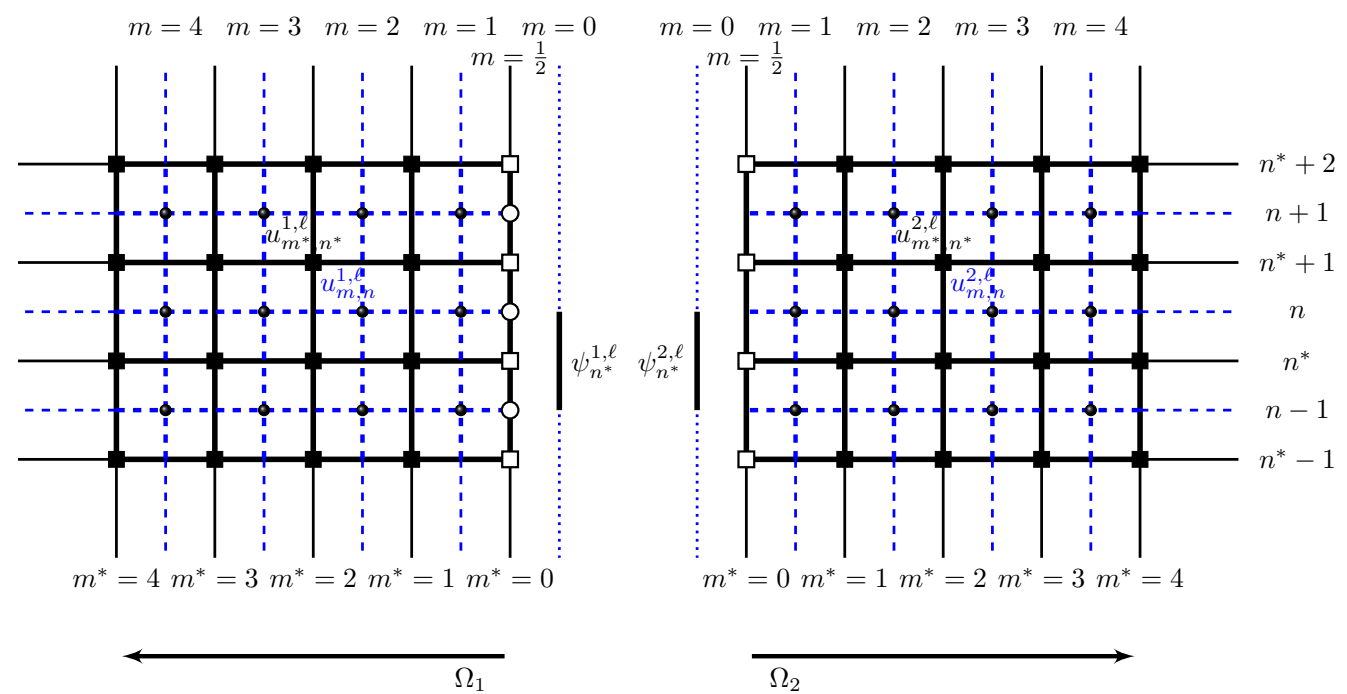

Figure 4: Notation for the rectangular DDFV configuration.

conditions, and for simplicity we still call it $u_{m, n}^{j, \ell}$. At each iteration $\ell=1,2, \ldots$, in the left domain $\Omega_{j}$ the values in the primal cells and the values in the dual cells, denoted with a star $*$, are related by two recurrence relations. We assume here that $A_{x y}=0$, so that the two recurrence relations are decoupled, the study of the fully anisotropic case is substantially harder and will be tackled in future work. For $m \geq 1$ and $m^{*} \geq 1$, we have

$$
\begin{aligned}
\frac{A_{x x}}{h_{x}^{2}}\left(u_{m+1, n}^{j, \ell}-2 u_{m, n}^{j, \ell}+u_{m-1, n}^{j, \ell}\right)+\frac{A_{y y}}{h_{y}^{2}}\left(u_{m, n+1}^{j, \ell}-2 u_{m, n}^{j, \ell}+u_{m, n-1}^{j, \ell}\right)-\eta u_{m, n}^{j, \ell} & =0, \\
\frac{A_{x x}}{h_{x}^{2}}\left(u_{m^{*}+1, n^{*}}^{j, \ell}-2 u_{m^{*}, n^{*}}^{j, \ell}+u_{m^{*}-1, n^{*}}^{j, \ell}\right)+\frac{A_{y y}}{h_{y}^{2}}\left(u_{m^{*}, n^{*}+1}^{j, \ell}-2 u_{m^{*}, n^{*}}^{j, \ell}+u_{m^{*}, n^{*}-1}^{j, \ell}-\eta u_{m^{*}, n^{*}}^{j, \ell}\right. & =0 .
\end{aligned}
$$

In order to obtain the primal equation in (3.1) for $m=1$, we introduce $u_{0, n}^{j, \ell}$ which is linked to the interface primal unknowns $u_{\frac{1}{2}, n}^{j, \ell}$ by

$$
u_{\frac{1}{2}, n}^{j, \ell}=\frac{1}{2}\left(u_{1, n}^{j, \ell}+u_{0, n}^{j, \ell}\right) .
$$

On boundary dual cells, the additional fluxes $\psi_{n^{*}}^{j, \ell}$ are used, given by

$$
h_{y} \psi_{n^{*}}^{j, \ell}+\frac{h_{y} A_{x x}}{h_{x}}\left(u_{1^{*}, n^{*}}^{j, \ell}-u_{0^{*}, n^{*}}^{j, \ell}\right)+\frac{A_{y y} h_{x}}{2 h_{y}}\left(u_{0^{*}, n^{*}+1}^{j, \ell}-2 u_{0^{*}, n^{*}}^{j, \ell}+u_{0^{*}, n^{*}-1}^{j, \ell}\right)-\eta \frac{h_{x} h_{y}}{2} u_{0^{*}, n^{*}}^{1, \ell}=0 .
$$

We can now express the transmission condition on $\Gamma$ for $(j, i)=(1,2)$ or $(2,1)$. The Robin transmission conditions on $\Gamma$ for dual cells are expressed with the fluxes $\psi_{n^{*}}^{j, \ell}$, see $(2.7 \mathrm{f})$ and $(2.15 \mathrm{~b})$,

$$
\psi_{n^{*}}^{j, \ell}+p^{*} u_{0^{*}, n^{*}}^{j, \ell}=-\psi_{n^{*}}^{i, \ell-1}+p^{*} u_{0^{*}, n^{*}}^{i, \ell-1},
$$

and on primal cells the discrete Robin conditions are, see (2.7e) and (2.15a),

$$
\frac{2}{h_{x}} A_{x x}\left(u_{\frac{1}{2}, n}^{j, \ell}-u_{1, n}^{j, \ell}\right)+p u_{\frac{1}{2}, n}^{j, \ell}=\frac{2}{h_{x}} A_{x x}\left(u_{1, n}^{i, \ell-1}-u_{\frac{1}{2}, n}^{i, \ell-1}\right)+p u_{\frac{1}{2}, n}^{i, \ell-1} .
$$

The equations (3.1-3.5) completely describe the original Robin DDFV optimized Schwarz algorithm from Section 2 for the specific two subdomain decomposition. We see that in the case of Cartesian meshes with $A_{x y}=0$, the optimized Schwarz algorithm for the primal and the dual meshes are decoupled. For the primal unknowns, the interface is at $m=\frac{1}{2}$,

$$
\begin{gathered}
\frac{A_{x x}}{h_{x}^{2}}\left(u_{m+1, n}^{1, \ell}-2 u_{m, n}^{1, \ell}+u_{m-1, n}^{1, \ell}\right)+\frac{A_{y y}}{h_{y}^{2}}\left(u_{m, n+1}^{1, \ell}-2 u_{m, n}^{1, \ell}+u_{m, n-1}^{1, \ell}\right)-\eta u_{m, n}^{1, \ell}=0, \\
\frac{2}{h_{x}} A_{x x}\left(u_{\frac{1}{2}, n}^{j, \ell}-u_{1, n}^{j, \ell}\right)+p_{c c} u_{\frac{1}{2}, n}^{j, \ell}=\frac{2}{h_{x}} A_{x x}\left(u_{1, n}^{i, \ell-1}-u_{\frac{1}{2}, n}^{i, \ell-1}\right)+p_{c c} u_{\frac{1}{2}, n}^{i, \ell-1} .
\end{gathered}
$$


This corresponds to a discrete optimized Schwarz algorithm with Robin transmission conditions for a cell centered (CC) 5-point finite difference discretization of the anisotropic diffusion problem (2.1), with the interface through the middle of the cells, and we therefore use now $p_{c c} \equiv p$ for the optimization parameter. For the dual unknowns, the interface is at $m=0$,

$$
\begin{aligned}
\frac{A_{x x}}{h_{x}^{2}} & \left(u_{m^{*}+1, n^{*}}^{1, \ell}-2 u_{m^{*}, n^{*}}^{1, \ell}+u_{m^{*}-1, n^{*}}^{1, \ell}\right)+\frac{A_{y y}}{h_{y}^{2}}\left(u_{m^{*}, n^{*}+1}^{1, \ell}-2 u_{m^{*}, n^{*}}^{1, \ell}+u_{m^{*}, n^{*}-1}^{1, \ell}\right)-\eta u_{m^{*}, n^{*}}^{1, \ell}=0, \\
& \frac{A_{x x}}{h_{x}}\left(u_{0^{*}, n^{*}}^{j, \ell}-u_{1^{*}, n^{*}}^{j, \ell}-\frac{A_{y y} h_{x}}{2 h_{y}^{2}}\left(u_{0^{*}, n^{*}+1}^{j, \ell}-2 u_{0^{*}, n^{*}}^{j, \ell}+u_{0^{*}, n^{*}-1}^{j, \ell}\right)+\eta \frac{h_{x}}{2} u_{0^{*}, n^{*}}^{j, \ell}+p_{v c} u_{0^{*}, n^{*}}^{j,}\right. \\
= & -\frac{A_{x x}}{h_{x}}\left(u_{0^{*}, n^{*}}^{i, \ell-1}-u_{1^{*}, n^{*}}^{i, \ell-1}\right)+\frac{A_{y y} h_{x}}{2 h_{y}^{2}}\left(u_{0^{*}, n^{*}+1}^{i, \ell-1}-2 u_{0^{*}, n^{*}}^{i, \ell-1}+u_{0^{*}, n^{*}-1}^{i, \ell-1}\right)-\eta \frac{h_{x}}{2} u_{0^{*}, n^{*}}^{i, \ell-1}+p_{v c} u_{0^{*}, n^{*}}^{i, \ell} .
\end{aligned}
$$

This is also a discrete optimized Schwarz algorithm with Robin transmission conditions, but for a vertex centered (VC) 5-point finite difference discretization of the anisotropic diffusion equation (2.1), with the interface on the boundary of the cells, and we therefore use now $p_{v c} \equiv p^{*}$ for the optimization parameter. The energy estimate convergence proof in Section 2 implies that each of these algorithms separately is convergent. In contrast to the common continuous approach (a few exceptions are [28, 33, 34] and [22]), we optimize parameters in the transmission conditions at the discrete level here, and we start with a separate analysis for the primal (CC) and the dual (VC) components of the DDFV optimized Schwarz algorithm, before tackling the coupled problem with $p_{v c}=p_{c c}$, which will need a new theoretical result on best approximation problems.

\subsection{Discrete Convergence Factor}

Since the domain is bounded in the $y$ direction, $y \in(0, b)$ with homogeneous Dirichlet boundary conditions, that is in the index $n$ and $n^{*}$, we use a discrete sine series expansion in the $y$ variable to compute the convergence factor for each sine mode, and study the existence and uniqueness of a set of best transmission parameters $p_{v c}$ and $p_{c c}$ that are minimizing the convergence factor over a given set of frequencies.

We expand for $n \in\{1, \ldots, N\}$ and $m \in\{1, \ldots, M\}$ with $N h_{y}=b$ the grid function $u_{m, n}$ in a discrete Fourier sine series,

$$
u_{m, n}=\sum_{k=1}^{k_{\max }} \hat{u}_{m}(k) \sin \left(k n \pi \frac{h_{y}}{b}\right),
$$

with a slightly different $k_{\max }$ depending on if the scheme is primal or dual, since the number of gridpoints differs by one,

$$
k_{\text {max }}:= \begin{cases}\frac{b}{h_{y}}-1 & \text { for the dual scheme } \\ \frac{b}{h_{y}} & \text { for the primal scheme. }\end{cases}
$$

The Fourier coefficients are functions of the variable $k: u_{m, n}^{j, \ell} \leftrightarrow \hat{u}_{m}^{j, \ell}(k)$ and $u_{m^{*}, n^{*}}^{j, \ell} \leftrightarrow \hat{u}_{m^{*}}^{j, \ell}(k)$, which is discrete in this case, but we will optimize over a continuous set for $k$ to simplify the analysis, since this makes a negligible difference for the resulting parameters [28, Subsection 4.2]. Introducing the Fourier sine expansion into the difference equation satisfied by $u_{m, n}$, we obtain by a direct calculation

$$
\frac{A_{y y}}{h_{y}^{2}}\left(u_{m, n+1}-2 u_{m, n}+u_{m, n-1}\right)-\eta u_{m, n}=-\sum_{k}\left(4 \frac{A_{y y}}{h_{y}^{2}} \sin ^{2}\left(\frac{k \pi h_{y}}{2 b}\right)+\eta\right) \hat{u}_{m}(k) \sin \left(k n \pi \frac{h_{y}}{b}\right) .
$$

To simplify the notation, we define the quantities

$$
\alpha(k):=\frac{4 A_{y y}}{h_{y}^{2}} \sin ^{2}\left(\frac{k \pi h_{y}}{2 b}\right), \quad \mu(k):=\frac{h_{x}^{2}}{A_{x x}}(\alpha(k)+\eta) .
$$

Then the discrete grid function $u_{m, n}$ is a solution of either of the two equations (3.1) if and only if the corresponding Fourier coefficients $\hat{u}_{m}(k)$ satisfy for each $k$ the recurrence relation in the $m$ variable

$$
\hat{u}_{m+1}(k)-2 \hat{u}_{m}(k)+\hat{u}_{m-1}(k)-\mu \hat{u}_{m}(k)=0 .
$$

The characteristic equation of the recurrence relation,

$$
\lambda^{2}-2 \lambda+1-\mu \lambda=0,
$$


has two positive distinct roots, which are inverses of each other. For fixed $k$, denoting by $\lambda(k)$ the root that is smaller than 1 ,

$$
\lambda(k):=1+\frac{\mu(k)}{2}-\sqrt{\mu(k)+\frac{\mu(k)^{2}}{4}}<1,
$$

the grid function $u_{m, n}$ is a solution of either of the two equations in (3.1) if and only if $\hat{u}_{m}(k)$ is a linear combination of $\lambda(k)^{m}$ and $\lambda(k)^{-m}$. We rewrite now the transmission conditions on the Fourier coefficients. On the primal cells we obtain

$$
\frac{A_{x x}}{h_{x}}\left(\hat{u}_{0}^{j, \ell}-\hat{u}_{1}^{j, \ell}\right)+\frac{p_{c c}}{2}\left(\hat{u}_{0}^{j, \ell}+\hat{u}_{1}^{j, \ell}\right)=\frac{A_{x x}}{h_{x}}\left(\hat{u}_{1}^{i, \ell-1}-\hat{u}_{0}^{i, \ell-1}\right)+\frac{p_{c c}}{2}\left(\hat{u}_{1}^{i, \ell-1}+\hat{u}_{0}^{i, \ell-1}\right) .
$$

and on the dual cells we obtain

$\frac{A_{x x}}{h_{x}}\left(\hat{u}_{0^{*}}^{j, \ell}-\hat{u}_{1^{*}}^{j, \ell}\right)+\frac{h_{x}}{2}(\alpha(k)+\eta) \hat{u}_{0^{*}}^{j, \ell}+p_{v c} \hat{u}_{0^{*}}^{j, \ell}=-\frac{A_{x x}}{h_{x}}\left(\hat{u}_{0^{*}}^{i, \ell-1}-\hat{u}_{1^{*}}^{i, \ell-1}\right)-\frac{h_{x}}{2}(\alpha(k)+\eta) \hat{u}_{0^{*}}^{i, \ell-1}+p_{v c} \hat{u}_{0^{*}}^{i, \ell-1}$.

Introducing the notation $\tilde{p}:=p \frac{h_{x}}{A_{x x}}$, we obtain for the system of transmission conditions in Fourier

$$
\begin{gathered}
\left(\hat{u}_{0}^{j, \ell}-\hat{u}_{1}^{j, \ell}\right)+\frac{\tilde{p}_{c c}}{2}\left(\hat{u}_{0}^{j, \ell}+\hat{u}_{1}^{j, \ell}\right)=\left(\hat{u}_{1}^{i, \ell-1}-\hat{u}_{0}^{i, \ell-1}\right)+\frac{\tilde{p}_{c c}}{2}\left(\hat{u}_{1}^{i, \ell-1}+\hat{u}_{0}^{i, \ell-1}\right), \\
\hat{u}_{0^{*}}^{j, \ell}-\hat{u}_{1^{*}}^{j, \ell}+\frac{\mu}{2} \hat{u}_{0^{*}}^{j, \ell}+\tilde{p}_{v c} \hat{u}_{0^{*}}^{j, \ell}=-\left(\hat{u}_{0^{*}}^{i, \ell-1}-\hat{u}_{1^{i}}^{i, \ell-1}\right)-\frac{\mu}{2} \hat{u}_{0^{*}}^{i, \ell-1}+\tilde{p}_{v c} \hat{u}_{0^{*}}^{i, \ell-1} .
\end{gathered}
$$

\subsection{Unbounded domain in the $x$ direction}

We start by considering an unbounded domain in the $x$ direction, $\Omega=(-a, a) \times(0, b)$ with $a=\infty$. By Parseval's relation, for $u_{m, n}^{j, \ell}$ to be in $L^{2}\left(\mathbb{R}_{ \pm} \times[0, b]\right)$ in $(x, y)$ for any $j, \ell$, there exist coefficients $C^{j, \ell}(k)$ and $D^{j, \ell}(k)$ such that

$$
\hat{u}_{m, k}^{j, \ell}=C^{j, \ell}(k) \lambda(k)^{m}, \quad \hat{u}_{m^{*}, k}^{j, \ell}=D^{j, \ell}(k) \lambda(k)^{m^{*}} .
$$

Inserting this into the transmission conditions (3.14) we obtain for $j=1,2, i \neq j$ and $l \geq 1$,

$$
\begin{array}{r}
\left(1-\lambda+\frac{\tilde{p}_{c c}}{2}(1+\lambda)\right) C^{j, \ell}=\left(-(1-\lambda)+\frac{\tilde{p}_{c c}}{2}(1+\lambda)\right) C^{i, \ell-1}, \\
\left(1-\lambda+\frac{\mu}{2}+\tilde{p}_{v c}\right) D^{j, \ell}=\left(-(1-\lambda)-\frac{\mu}{2}+\tilde{p}_{v c}\right) D^{i, \ell-1} .
\end{array}
$$

We now introduce the notation

$$
\nu(k):=-\ln \lambda(k)>0,
$$

which lets us write the transmission conditions in Fourier in compact form: solving (3.12) for $\mu$ and introducing it into the following expression, we find

$$
1+\frac{\mu}{2}-\lambda=\frac{1-\lambda^{2}}{2 \lambda}=\sinh \nu \quad \text { and } \quad \frac{1-\lambda}{1+\lambda}=\tanh \frac{\nu}{2},
$$

which gives for the transmission conditions

$$
\begin{gathered}
\left(2 \tanh \frac{\nu}{2}+\tilde{p}_{c c}\right) C^{j, \ell}=\left(-2 \tanh \frac{\nu}{2}+\tilde{p}_{c c}\right) C^{i, \ell-1}, \\
\left(\sinh \nu+\tilde{p}_{v c}\right) D^{j, \ell}=\left(-\sinh \nu+\tilde{p}_{v c}\right) D^{i, \ell-1} .
\end{gathered}
$$

Writing this iteration in matrix form over two iteration steps, we obtain

$$
\left(\begin{array}{c}
C^{j, \ell} \\
D^{j, \ell}
\end{array}\right)=R_{d, \infty}\left(p_{c c}, p_{v c}, \nu\right)\left(\begin{array}{c}
C^{j, \ell-2} \\
D^{j, \ell-2}
\end{array}\right), \quad R_{d, \infty}\left(p_{c c}, p_{v c}, \nu\right):=\left(\begin{array}{cc}
\rho_{c c, \infty}\left(p_{c c}, \nu\right) & 0 \\
0 & \rho_{v c, \infty}\left(p_{v c}, \nu\right)
\end{array}\right),
$$

with the convergence factors of the vertex centered and cell centered schemes given by

$$
\begin{aligned}
\rho_{c c, \infty}(p, \nu) & :=\frac{p-f_{c c, \infty}(\nu)}{p+f_{c c, \infty}(\nu)}, & \rho_{v c, \infty}(p, \nu) & :=\frac{p-f_{v c, \infty}(\nu)}{p+f_{v c, \infty}(\nu)} \\
f_{c c, \infty}(\nu) & :=2 \frac{A_{x x}}{h_{x}} \tanh \frac{\nu}{2}, & f_{v c, \infty}(\nu) & :=\frac{A_{x x}}{h_{x}} \sinh \nu .
\end{aligned}
$$




\subsection{Bounded domain in the $x$ direction}

Outer boundary conditions can have an influence on the convergence of Schwarz methods, see for example [14], and we therefore now also study the case of a bounded domain, $\Omega=(-a, a) \times(0, b)$ with $a=M h_{x}=$ $M^{*} h_{x}<\infty$, which leads to slightly more complicated formulas. The Fourier coefficients of the sine transformed grid function are then of the form

$$
\hat{u}_{m, k}^{j, \ell}=C^{j, \ell}(k) \lambda(k)^{m}+\tilde{C}^{j, \ell}(k) \lambda(k)^{-m}, \quad \hat{u}_{m^{*}, k}^{j, \ell}=D^{j, \ell}(k) \lambda(k)^{m^{*}}+\tilde{D}^{j, \ell}(k) \lambda(k)^{-m^{*}} .
$$

With the notations in Figure 4, the Dirichlet boundary conditions are enforced in both subdomains as

$$
u_{M^{*}, n}=0, \quad \frac{1}{2}\left(u_{M, n}+u_{M+1, n}\right)=0 .
$$

This gives the relations between the coefficients $C, D$ and their tilde counterpart,

$$
\tilde{C}^{j, \ell}(k)=-\lambda^{2 M+1} C^{j, \ell}(k), \quad \tilde{D}^{j, \ell}(k)=-\lambda^{2 M} D^{j, \ell}(k),
$$

which leads to the Fourier coefficients

$$
\hat{u}_{m, k}^{j, \ell}=C^{j, \ell}(k)\left(\lambda(k)^{m}-\lambda(k)^{2 M+1-m}\right), \quad \hat{u}_{m^{*}, k}^{j, \ell}=D^{j, \ell}(k)\left(\lambda(k)^{m^{*}}-\lambda(k)^{2 M-m^{*}}\right) .
$$

Inserting these expressions into the transmission conditions (3.14) we get after simplification using again

$$
\begin{aligned}
\left(2 \tanh \left(\frac{\nu}{2}\right) \operatorname{coth}(M \nu)+\tilde{p}_{c c}\right) C^{j, \ell} & =\left(-2 \tanh \left(\frac{\nu}{2} \operatorname{coth}(M \nu)+\tilde{p}_{c c}\right) C^{i, \ell-1}\right. \\
\left(\sinh (\nu) \operatorname{coth}(M \nu)+\tilde{p}_{v c}\right) D^{j, \ell} & =\left(-\sinh (\nu) \operatorname{coth}(M \nu)+\tilde{p}_{v c}\right) D^{i, \ell-1}
\end{aligned}
$$

We thus also get a matrix iteration similar to the unbounded case in (3.18), namely

$$
\left(\begin{array}{c}
C^{j, \ell} \\
D^{j, \ell}
\end{array}\right)=R_{d, M}\left(p_{c c, M}, p_{v c, M}, \nu\right)\left(\begin{array}{l}
C^{j, \ell-2} \\
D^{j, \ell-2}
\end{array}\right), R_{d, M}\left(p_{c c, M}, p_{v c, M}, \nu\right):=\left(\begin{array}{cc}
\rho_{c c, M}\left(p_{c c, M}, \nu\right) & 0 \\
0 & \rho_{v c, M}\left(p_{v c, M}, \nu\right)
\end{array}\right),
$$

with the convergence factors of the vertex centered and cell centered schemes for the bounded case given by

$$
\begin{aligned}
\rho_{c c, M}(p, \nu) & =\frac{p-f_{c c, M}(\nu)}{p+f_{c c, M}(\nu)}, & \rho_{v c, M}(p, \nu) & =\frac{p-f_{v c, M}(\nu)}{p+f_{v c, M}(\nu)}, \\
f_{c c, M}(\nu) & =2 \frac{A_{x x}}{h_{x}} \tanh \frac{\nu}{2} \operatorname{coth}(M \nu), & f_{v c, M}(\nu) & =\frac{A_{x x}}{h_{x}} \sinh \nu \operatorname{coth}(M \nu) .
\end{aligned}
$$

We now recall the convergence factors for the continuous algorithm from [17],

$$
\begin{aligned}
\rho_{c, a}(p, r) & =\frac{p-f_{c, a}(r)}{p+f_{c, a}(r)}, & \rho_{c, \infty}(p, r) & =\frac{p-f_{c, \infty}(r)}{p+f_{c, \infty}(r)} \\
f_{c, a}(r) & =A_{x x} r \operatorname{coth}(a r), & f_{c, \infty}(r) & =A_{x x} r
\end{aligned}
$$

where

$$
r(k):=\frac{1}{A_{x x}} \sqrt{\eta A_{x x}+\left(\frac{\pi k}{b}\right)^{2} \operatorname{det} A} .
$$

It is because we introduced the quantity $\nu(k)$ in (3.16) that the discrete convergence factors have a very similar form to the continuous ones from [17], only the functions $f$ which are summarized in Table 1 change. For small $h_{x}$ and $h_{y}, \nu$ is equivalent to $r h_{x}$, and for $a=M h_{x}$, ar is equivalent to $M \nu$. The functions for the continuous and discrete problems are equivalent at first order. We need to find parameters $p$ in the transmission conditions which minimize the convergence factors $\rho(k)$ in modulus over all frequencies $k$, and we investigate two options: the first is to use two parameters $p_{c c}$ and $p_{v c}$ and optimize separately the convergence factors $\rho_{c c}$ for the primal and $\rho_{v c}$ for the dual equation. The second, which is a first step toward the fully anisotropic problem, is to use $p_{v c}=p_{c c}$, and to minimize the spectral radius of the iteration matrix $R(k)$ for all $k$. 


\begin{tabular}{|l|l|l|}
\hline \multicolumn{1}{|c|}{ Continuous } & \multicolumn{1}{|c|}{ Discrete CC } & \multicolumn{1}{c|}{ Discrete VC } \\
\hline$f_{c, \infty}(r)=A_{x x} r$ & $f_{c c, \infty}(\nu)=2 \frac{A_{x x}}{h_{x}} \tanh \frac{\nu}{2}$ & $f_{v c, \infty}(\nu)=\frac{A_{x x}}{h_{x}} \sinh \nu$ \\
\hline$f_{c, a}(r)=A_{x x} r \operatorname{coth}(a r)$ & $f_{c c, M}(\nu)=2 \frac{A_{x x}}{h_{x}} \tanh \frac{\nu}{2} \operatorname{coth}(M \nu)$ & $f_{v c, M}(\nu)=\frac{A_{x x}}{h_{x}} \sinh \nu \operatorname{coth}(M \nu)$ \\
\hline$r=\frac{1}{A_{x x}} \sqrt{\eta A_{x x}+\left(\frac{\pi k}{b}\right)^{2} \operatorname{det} A}$ & $\nu=-\log \left(1+\frac{\mu(k)}{2}-\sqrt{\mu(k)+\frac{\mu(k)^{2}}{4}}\right), \mu(k):=\frac{h_{x}^{2}}{A_{x x}}\left(\frac{4 A_{y y}}{h_{y}^{2}} \sin ^{2}\left(\frac{k \pi h_{y}}{2 b}\right)+\eta\right)$ \\
\hline
\end{tabular}

Table 1: Functions involved in the various convergence factors

\subsection{The best approximation problem: separate optimization}

Since with our change of variables (3.16) all convergence factors are of the same form, we define a general minmax problem for a continuous function $f$ of $s$ on $K=\left[s_{\min }, s_{\max }\right]$,

$$
\begin{gathered}
\text { for } F(p, s):=\frac{p-f(s)}{p+f(s)} \text { and } G(p):=\sup _{s \in K}|F(p, s)| \\
\text { find } p^{\text {opt }} \in \mathbb{R} \text { such that } G\left(p^{o p t}\right)=\inf _{p \in \mathbb{R}} G(p):=\delta^{o p t} .
\end{gathered}
$$

Following ideas in [12], we now solve this minmax problem for rather general functions $f$ with the help of several lemmas.

Lemma 3.1. If $f$ is the identity function, then problem (3.24) has a unique solution, given by

$$
p^{o p t}=\sqrt{s_{\min } s_{\max }}, \delta^{o p t}=F\left(p^{o p t}, s_{\min }\right)=-F\left(p^{o p t}, s_{\max }\right) .
$$

Proof. If $p$ is outside $K$, then moving $p$ toward $K$ decreases $F$ uniformly, so the best $p$ must be in $K$. But then $G(p)=\max \left(\left|F\left(p, s_{\min }\right)\right|,\left|F\left(p, s_{\max }\right)\right|\right)$, and the minimum of $G$ is thus attained when $F\left(p, s_{\min }\right)=-F\left(p, s_{\max }\right)$, which gives $(3.25)$.

Lemma 3.2. If $f$ is a positive monotonic function, then Problem (3.24) has a unique solution, given by

$$
p^{o p t}=\sqrt{f\left(s_{\min }\right) f\left(s_{\max }\right)}, \delta^{o p t}=F\left(p^{o p t}, s_{\min }\right)=-F\left(p^{o p t}, s_{\max }\right) .
$$

Proof. This proof is obtained from Lemma 3.1 by the bijective change of variables $f$.

Lemma 3.3. Both for the unbounded and bounded domain case, the functions $f(r)$ associated with the continuous convergence factors, and $f(\nu)$ associated with the discrete cell centered and vertex centered convergence factors, are positive monotonic functions.

Proof. For the unbounded case, we see from the first line in Table 1 that the functions are increasing. For the bounded case, for $f_{c, a}$, we only need to check that the function $s \operatorname{coth} s$ is increasing, which we see directly by writing its derivative in the form $\frac{\sinh 2 s-2 s}{2 \sinh ^{2} s}>0$. For $f_{v c, M}$, we differentiate in $\nu$ to find

$$
f_{v c, M}^{\prime}(\nu)=\frac{A_{x x}}{h_{x}} \frac{\frac{1}{2} \cosh \nu \sinh (2 M \nu)-M \sinh \nu}{\sinh ^{2}(M \nu)} .
$$

Differentiating the numerator again we find

$$
M \cosh \nu(\cosh (2 M \nu)-1)+\frac{1}{2} \sinh \nu \sinh (2 M \nu)>0,
$$

which shows that the numerator of $f_{v c, M}^{\prime}(\nu)$ is increasing, and since it is zero for $\nu=0$, it must be positive. Hence the function $f_{v c, M}$ is increasing. For $f_{c c, M}$, its derivative is

$$
f_{c c, M}^{\prime}(\nu)=\frac{A_{x x}}{h_{x}} \frac{\sinh (2 M \nu)-2 M \sinh \nu}{2 \sinh ^{2}(M \nu) \cosh ^{2} \nu} .
$$

A series expansion of the numerator shows that this quantity is positive for $M \geq 1$,

$$
\sinh (2 M \nu)-2 M \sinh \nu=\sum_{n \geq 1} 2 M\left((2 M)^{2 n}-1\right) \frac{\nu^{2 n+1}}{(2 n+1) !}>0,
$$

which concludes the proof of the lemma. 
It remains to apply the lemmas to the functions $f$ for the different cases to obtain

Theorem 3.1. The best performance of the Robin Schwarz algorithm both in the unbounded and bounded domains, in the continuous case with $s:=r$ and in the discrete cases with $s:=\nu$ is attained for $p^{o p t}=\sqrt{f\left(s\left(k_{\min }\right)\right) f\left(s\left(k_{\max }\right)\right)}$, where $f$ is given in Table 1, and the associated convergence factor is then bounded by $\left|\frac{\sqrt{f\left(s\left(k_{\max }\right)\right)}-\sqrt{f\left(s\left(k_{\min }\right)\right)}}{\sqrt{f\left(s\left(k_{\max }\right)\right)}+\sqrt{f\left(s\left(k_{\min }\right)\right)}}\right|$. Here, $k_{\min }=1$ and $k_{\max }$ is defined in (3.8).

\subsection{Coupled Optimization}

We suppose now that the optimization parameters $p_{c c}$ and $p_{v c}$ for the primal and dual problems are equal to $p, p_{c c}=p_{v c}=p$. The convergence speed is then governed both in the bounded and unbounded case by the spectral radius of the corresponding iteration matrix $R$, that is

$$
\rho_{d}(p, \nu):=\max \left(\left|\rho_{c c}(p, \nu)\right|,\left|\rho_{v c}(p, \nu)\right|\right) \text {. }
$$

We thus obtain for two functions $f_{1}\left(=f_{c c}\right)$ and $f_{2}\left(=f_{v c}\right)$ the general best approximation problem

$$
\begin{aligned}
& \text { for } F_{j}(p, \nu)=\frac{p-f_{j}(\nu)}{p+f_{j}(\nu)}, F(p, \nu)=\max _{j}\left(\left|F_{j}(p, \nu)\right|\right), \quad G(p)=\sup _{\nu \in K}|F(p, \nu)|, \\
& \text { find } p^{o p t} \in \mathbb{R}, \quad G\left(p^{o p t}\right)=\inf _{p \in \mathbb{R}} G(p)=: \delta^{o p t}, \quad K:=\left[\nu_{\min }, \nu_{\max }\right] .
\end{aligned}
$$

Lemma 3.4. If $f_{1}$ and $f_{2}$ are positive increasing functions of $\nu$, and $f_{2} \geq f_{1}$, then there exists a unique solution popt of (3.26) given by

$$
p^{o p t}=\sqrt{f_{1}\left(\nu_{\min }\right) f_{2}\left(\nu_{\max }\right)}, \quad \delta^{o p t}=\frac{\sqrt{f_{2}\left(\nu_{\max }\right)}-\sqrt{f_{1}\left(\nu_{\min }\right)}}{\sqrt{f_{2}\left(\nu_{\max }\right)}+\sqrt{f_{1}\left(\nu_{\min }\right)}} .
$$

Proof. Since $\delta^{\text {opt }} \leq G(p=1)<1$, popt must be positive, since $G(p<0)>1$. We next evaluate the maximum in the function $F$ : for positive $p$, we obtain

$$
\left(\frac{p-f_{1}(\nu)}{p+f_{1}(\nu)}\right)^{2}-\left(\frac{p-f_{2}(\nu)}{p+f_{2}(\nu)}\right)^{2}=\frac{4 p\left(f_{2}(\nu)-f_{1}(\nu)\right)\left(p^{2}-f_{1}(\nu) f_{2}(\nu)\right)}{\left(p+f_{1}(\nu)\right)^{2}\left(p+f_{2}(\nu)\right)^{2}}
$$

which shows that

$$
F(p, \nu)= \begin{cases}\left|\frac{f_{2}(\nu)-p}{f_{2}(\nu)+p}\right| & \text { if } p \leq \sqrt{f_{1}(\nu) f_{2}(\nu)}, \\ \frac{f_{1}(\nu)-p}{f_{1}(\nu)+p} \mid & \text { if } p \geq \sqrt{f_{1}(\nu) f_{2}(\nu)} .\end{cases}
$$

Now for all $\nu$, we have by assumption that $f_{2}(\nu) \geq f_{1}(\nu)$, and therefore $p \leq \sqrt{f_{1}(\nu) f_{2}(\nu)}$ implies $p \leq f_{2}(\nu)$, and $p \geq \sqrt{f_{1}(\nu) f_{2}(\nu)}$ implies $p \geq f_{1}(\nu)$. The function $f$ defined by $f(\nu)=\sqrt{f_{1}(\nu) f_{2}(\nu)}$ is increasing therefore bijective, and we can thus write $F$ from (3.28) without the modulus,

$$
F(p, \nu)= \begin{cases}\frac{f_{2}(\nu)-p}{f_{2}(\nu)+p} & \text { if } p \leq f(\nu) \\ \frac{p-f_{1}(\nu)}{f_{1}(\nu)+p} & \text { if } p \geq f(\nu)\end{cases}
$$

We next show that if $p^{\text {opt }}$ exists, it must lie in $f(K)$ : if $p \leq f\left(\nu_{\min }\right)$ then for all $\nu \in K, p \leq f(\nu)$, and therefore

$$
F(p, \nu)=\frac{f_{2}(\nu)-p}{f_{2}(\nu)+p}
$$

which is an increasing function of $\nu$ over $K$, and therefore reaches its maximum at $\nu_{\text {max }}$ :

$$
G(p)=F\left(p, \nu_{\max }\right)=\frac{f_{2}\left(\nu_{\max }\right)-p}{f_{2}\left(\nu_{\max }\right)+p} .
$$


Now $G$ is a continuous decreasing function of $p$ on $\left(-\infty, f\left(\nu_{\min }\right)\right)$ and reaches its minimum at $f\left(\nu_{\min }\right)$. A similar argument holds for $p \geq f\left(\nu_{\max }\right)$ and $\left(f\left(\nu_{\max }\right),+\infty\right)$. Therefore the minimum of $G$ over $\mathbb{R}$ is reached over $f(K)$. Then by compactness and continuity, there exists $p^{o p t} \in f(K), \delta^{o p t} \in(0,1)$ solution of the minmax problem.

To determine $p^{o p t} \in f(K)$, we consider $F$ now as a function of $\nu$,

$$
F(p, \nu)= \begin{cases}\frac{p-f_{1}(\nu)}{f_{1}(\nu)+p} & \text { if } \nu \leq f^{-1}(p), \\ \frac{f_{2}(\nu)-p}{f_{2}(\nu)+p} & \text { if } \nu \geq f^{-1}(p) .\end{cases}
$$

Hence $F(p, \nu)$ is decreasing in $\nu$ on $\left[\nu_{\min }, f^{-1}(p)\right]$, and increasing in $\nu$ on $\left[f^{-1}(p), \nu_{\max }\right]$, and therefore

$$
G(p)=\max \left(g_{1}(p), g_{2}(p)\right) \text { with } g_{1}(p)=\frac{p-f_{1}\left(\nu_{\min }\right)}{f_{1}\left(\nu_{\min }\right)+p} \text { and } g_{2}(p)=\frac{f_{2}\left(\nu_{\max }\right)-p}{f_{2}\left(\nu_{\max }\right)+p} .
$$

As $g_{1}$ increases, $g_{2}$ decreases, and looking at their values at 0 and $\infty$, it easy to see that there exists $p^{\text {opt }}$ such that $G(p)=g_{2}(p)$ for $p \leq p^{\text {opt }}$ and $G(p)=g_{1}(p)$ for $p \geq p^{o p t}$. Hence, the minimum is reached at $p^{o p t}$, that is at equilibrium, $g_{1}\left(p^{o p t}\right)=g_{2}\left(p^{o p t}\right)$, and $p^{o p t}=\sqrt{f_{1}\left(\nu_{\min }\right) f_{2}\left(\nu_{\max }\right)}$, which leads to

$$
G\left(p^{o p t}\right)=\frac{\sqrt{f_{2}\left(\nu_{\max }\right)}-\sqrt{f_{1}\left(\nu_{\min }\right)}}{\sqrt{f_{2}\left(\nu_{\max }\right)}+\sqrt{f_{1}\left(\nu_{\min }\right)}} .
$$

Theorem 3.2. The best performance for the DDFV Robin Schwarz algorithm for a single parameter $p_{c c}=p_{v c}=p$ is obtained for

$$
p_{d d f v, \alpha}^{o p t}=\sqrt{f_{c c, \alpha}\left(\nu_{\min }\right) f_{v c, \alpha}\left(\nu_{\max }\right)},
$$

where $\alpha \in\{M, \infty\}$ for either the bounded or unbounded case from Table 1, and the convergence factor is then bounded by $\frac{\sqrt{f_{v c, \alpha}\left(\nu_{\max }\right)}-\sqrt{f_{c c, \alpha}\left(\nu_{\min }\right)}}{\sqrt{f_{v c, \alpha}\left(\nu_{\max }\right)}+\sqrt{f_{c c, \alpha}\left(\nu_{\min }\right)}}$, where $\nu_{\min }=\nu\left(k_{\min }\right), \nu_{\max }=\nu\left(k_{\max }\right)$ with $k_{\min }=1$ and $k_{\max }=\frac{b}{h_{y}}-1$.

Proof. It suffices to notice that for all $\nu>0, f_{c c, \alpha}(\nu)<f_{v c, \alpha}(\nu)$, as we can see from Table 1, since

$$
f_{c c, \alpha}(\nu)<f_{v c, \alpha}(\nu) \Longleftrightarrow 2 \tanh \frac{\nu}{2}<\sinh \nu \Longleftrightarrow 2 \frac{\sinh \frac{\nu}{2}}{\cosh \frac{\nu}{2}}<2 \sinh \frac{\nu}{2} \cosh \frac{\nu}{2} \Longleftrightarrow \cosh ^{2} \frac{\nu}{2}>1,
$$

which clearly holds. Then apply Lemma 3.4 with $f_{1}=f_{c c, \alpha}$ and $f_{2}=f_{v c, \alpha}$.

\section{Numerical Experiments}

We now test our optimized DDFV Schwarz algorithms numerically, both for cases covered by our analysis on rectangular meshes with two subdomain decompositions, and more general meshes and decompositions including cross points.

\subsection{Experiments Covered by our Analysis}

We study as our first model problem

$$
-\nabla \cdot(A \nabla u)+u=0, \quad \text { in } \Omega=(-1,1) \times(0,1),
$$

with the two subdomains $\Omega_{1}=(-1,0) \times(0,1)$ and $\Omega_{1}=(0,1) \times(0,1)$, and use rectangular meshes, for which our fully discrete analysis holds. We determine numerically optimized parameters $p^{\text {num }}$ by running our implementation to find the parameter which gives the best performance. We simulate directly the 


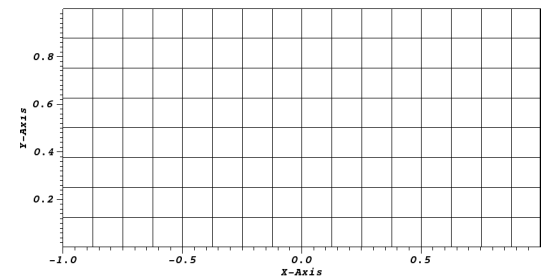

$x-A \times i s$

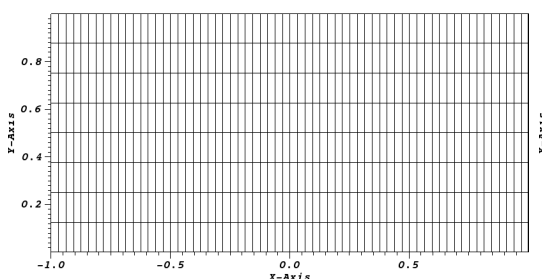

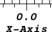

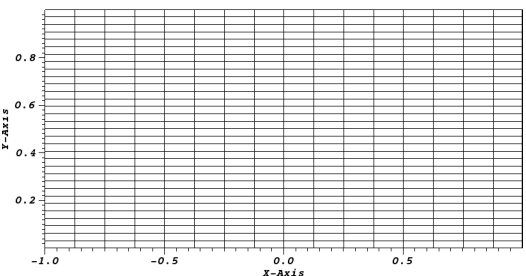

0.0

Figure 5: Isotropic rectangular mesh called $\mathrm{m} 22$ on the left, and anisotropically refined rectangular mesh in the $x$-direction called anisox $4 \mathrm{y} 1$ in the middle, and in the $y$-direction called anisox $1 \mathrm{y} 4$ on the right, each for the corresponding second lines in Table 2.

\begin{tabular}{|c|c|c|c|c|c|c|c|c|c|c|c|c|c|}
\hline & \multicolumn{4}{|c|}{ Continuous Analysis } & \multicolumn{6}{|c|}{ Discrete Analysis } & \multicolumn{3}{|c|}{ Numerically Best } \\
\hline \multirow[t]{2}{*}{$\Delta x, \Delta y$} & $p_{c c c, \infty}^{o p t}$ & $p_{c v c,}^{o p t}$ & $p_{c c c, a}^{o p t}$ & $p_{c v c, a}^{o p t}$ & $p_{c c, \infty}^{o p t}$ & $p_{v c, \infty}^{o p t}$ & $p_{c c, M}^{o p t}$ & $p_{v c, 1}^{o p t}$ & $p_{d d f \imath}^{o p t}$ & $a a_{j}$ & $p_{c c, n}^{o p t}$ & $p_{v c, n}^{o p t}$ & $a_{j}$ \\
\hline & \multicolumn{13}{|c|}{$A_{x x}=1, A_{y y}=1, \eta=1$, mesh $\mathrm{m} 22$} \\
\hline${ }^{-3}, 2^{-3}$ & 9.11 & 8.52 & 9.12 & 8.53 & 6.03 & 8.59 & 6.04 & 8.60 & 8.54 & 8.55 & 6.04 & 8.59 & 8.27 \\
\hline $2^{-4}, 2^{-4}$ & 12.87 & 12.47 & 12.89 & 12.48 & 8.61 & 12.20 & 8.62 & 12.22 & 12.18 & 12.20 & 8.61 & 12.18 & 11.79 \\
\hline $2^{-5}, 2^{-5}$ & 18.21 & 17.92 & 18.23 & 17.94 & 12.20 & 17.27 & 12.22 & 17.29 & 17.26 & 17.29 & 12.20 & 17.20 & 16.26 \\
\hline \multirow[t]{2}{*}{$2^{-6}, 2^{-6}$} & 25.75 & 25.54 & 25.78 & 25.58 & 17.27 & 24.43 & 17.29 & 24.46 & 24.42 & 24.46 & 17.24 & 24.32 & 22.13 \\
\hline & \multicolumn{13}{|c|}{$A_{x x}=16, A_{y y}=1, \eta=1$, mesh $\mathrm{m} 22$} \\
\hline $2^{-3}, 2^{-3}$ & 36.43 & 34.08 & 44.26 & 41.41 & 28.54 & 29.16 & 34.76 & 35.52 & 29.42 & 35.83 & 34.71 & 35.49 & 35.01 \\
\hline $2^{-4}, 2^{-4}$ & 51.50 & 49.86 & 62.57 & 60.59 & 40.44 & 41.59 & 49.16 & 50.56 & 41.69 & 50.68 & 49.11 & 50.48 & 49.59 \\
\hline $2^{-5}, 2^{-5}$ & 72.82 & 71.68 & 88.48 & 87.09 & 57.22 & 58.95 & 69.53 & 71.63 & 58.98 & 71.67 & 69.37 & 71.38 & 58.66 \\
\hline \multirow[t]{2}{*}{$2^{-6}, 2^{-6}$} & 102.99 & 102.18 & 125.13 & 124.15 & 80.93 & 83.41 & 98.34 & 101.35 & 83.42 & 101.36 & 98.02 & 100.94 & 96.45 \\
\hline & \multicolumn{13}{|c|}{$A_{x x}=16, A_{y y}=1, \eta=1$, mesh anisox1y 4} \\
\hline $2^{-5}$ & 72.82 & 71.68 & 88.48 & 87.09 & 48.82 & 69.07 & $\overline{59.33}$ & 83.95 & 69.05 & 83.91 & 59.23 & 83.57 & 80.07 \\
\hline $2^{-4}, 2^{-6}$ & 102.99 & 102.18 & 125.13 & 124.15 & 69.08 & 97.71 & 83.94 & 118.73 & 97.70 & 118.72 & 83.73 & 118.14 & 109.80 \\
\hline $2^{-5}, 2^{-7}$ & 145.64 & 145.07 & 176.96 & 176.27 & 97.71 & 138.19 & 118.73 & 167.91 & 138.19 & 167.91 & 118.20 & 166.69 & 148.52 \\
\hline \multirow[t]{2}{*}{$2^{-6}, 2^{-\varepsilon}$} & 205.97 & 205.57 & 250.2 & 249.77 & 138.19 & 195.44 & 167.91 & 237.46 & 195.43 & 237.46 & 167.15 & 235.69 & 192.65 \\
\hline & \multicolumn{13}{|c|}{$A_{x x}=1, A_{y y}=16, \eta=1$, mesh $\mathrm{m} 22$} \\
\hline $2^{-3}, 2^{-3}$ & 35.60 & 33.30 & 35.60 & 33.30 & 12.37 & 63.59 & 12.37 & 63.59 & 51.02 & 51.02 & 12.37 & 63.44 & 48.41 \\
\hline $2^{-4}, 2^{-4}$ & 50.35 & 48.75 & 50.35 & 48.75 & 19.07 & 84.09 & 19.07 & 84.09 & 78.62 & \begin{tabular}{|l|}
78.62 \\
\end{tabular} & 19.06 & 83.70 & 74.03 \\
\hline $2^{-5}, 2^{-5}$ & 71.20 & 70.08 & 71.20 & 70.08 & 27.71 & 116.29 & 27.71 & 116.29 & 114.24 & 114.24 & 27.68 & 115.37 & 103.04 \\
\hline \multirow[t]{2}{*}{$2^{-6}, 2^{-6}$} & 100.69 & 99.90 & 100.69 & 99.90 & 39.47 & 163.47 & 39.47 & 163.47 & 162.73 & 162.73 & 39.41 & 161.89 & 135.74 \\
\hline & \multicolumn{13}{|c|}{$A_{x x}=1, A_{y y}=16, \eta=1$, mesh anisox $4 \mathrm{y} 1$} \\
\hline $2^{-5}, 2^{-3}$ & 35.60 & 33.30 & 35.60 & 33.30 & 23.59 & 33.50 & 23.59 & 33.50 & \begin{tabular}{|l|l}
33.36 \\
\end{tabular} & 33.36 & 23.55 & \begin{tabular}{|l|}
33.45 \\
\end{tabular} & 32.25 \\
\hline $2^{-6}, 2^{-4}$ & 50.35 & 48.75 & 50.35 & 48.75 & 33.67 & 47.67 & 33.67 & 47.67 & 47.62 & 47.62 & 33.63 & 47.54 & 45.88 \\
\hline $2^{-7}, 2^{-5}$ & 71.20 & 70.08 & 71.20 & 70.08 & 47.73 & 67.52 & 47.73 & 67.52 & 67.50 & \begin{tabular}{|l|l|}
67.50 \\
\end{tabular} & 47.63 & 67.17 & 63.33 \\
\hline $2^{-8}, 2^{-6}$ & 100.69 & 99.90 & 100.69 & 99.90 & 67.54 & 95.53 & 67.54 & 95.53 & 95.52 & 95.52 & 67.32 & 94.96 & 85.93 \\
\hline
\end{tabular}

Table 2: Theoretically and numerically optimized parameters $p$ for various anisotropic diffusions and meshes. 

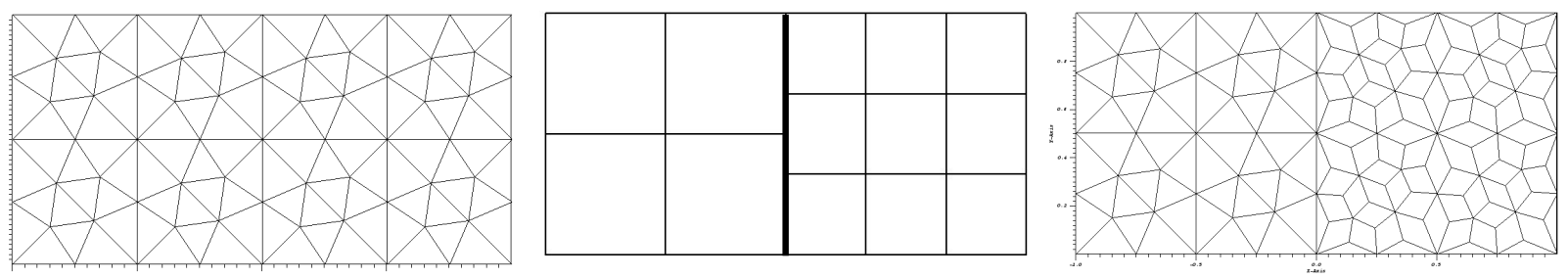

Figure 6: Meshes not covered by our discrete analysis used with refinements for Table 3.

error equations, and start with a random initial guess to make sure all error components are present. We use the three meshes shown in Figure 5, which we refine by dividing the mesh sizes by 2 several times. We show in Table 2 the corresponding results, including our theoretically optimized parameters at the continuous and discrete level from Theorem 3.1 and Theorem 3.2. For the cell centered and vertex centered schemes, we use for the optimized parameters the same notation as in Table 1 for the corresponding functions: $p_{c c, \infty}^{o p t}$ and $p_{v c, \infty}^{o p t}$ for the unbounded domain optimized choice, and $p_{c c, M}^{o p t}$ and $p_{v c, M}^{o p t}$ for the bounded domain optimized choice. For the DDFV results, we use $p_{d d f v, \infty}^{o p t}$ and $p_{d d f v, M}^{o p t}$ for the theoretical optimized parameters from Theorem 3.2. For the optimized parameters from the continuous analysis, there is also a small influence on the value depending on the use of a cell centered scheme or a vertex centered scheme, since the cell centered scheme uses one more grid point in the interior, which increases the estimate $k_{\max }$, see (3.8), and we use $p_{c c c, \infty}^{o p t}, p_{c v c, \infty}^{o p t}, p_{c c c, a}^{o p t}$, and $p_{c v c, a}^{o p t}$ for the corresponding values. Finally, we denote by $p_{c c, \text { num }}^{o p t}, p_{v c, \text { num }}^{o p t}$ and $p_{d d f v, \text { num }}^{\text {opt }}$ the value of the parameter which worked best in the numerical experiments by minimizing the numerical convergence factor $\rho_{\text {num }}$ of the method. The numerical convergence factors $\rho_{\text {num }}$ were computed by dividing the error after 100 iterations by the initial error and taking the 1/99-th root, and we denote the optimized value by $\rho_{\text {num }}^{\text {opt }}$. There are several interesting observations: first, we see that our new discrete bounded domain analysis very well predicts the numerical behavior, both for the cell centered and vertex centered discretizations and the combined DDFV scheme. Second, we see that for the Laplace case on an isotropic mesh, the bounded and unbounded analyses give similar results, and the vertex centered scheme performs like predicted also by the continuous analysis, while the cell centered scheme works best for a slightly smaller value of the parameter, which is very well captured by the discrete analysis. In the anisotropic case with isotropic mesh, there is a substantial difference between the continuous and discrete analysis, and the continuous parameters work less well with strong diffusion along the interface direction, $A_{y y}=16$. There is also a big difference between the cell centered and vertex centered discretizations then, the former needs a much smaller, and the latter a much larger optimized parameter than predicted by the continuous analysis. This is very well captured by our new discrete analysis. With strong diffusion across the interface, $A_{x x}=16$, the difference between the bounded and unbounded domain analysis becomes important, but the difference between cell centered and vertex centered discretization is negligible. Finally, if one adapts the discretization to the anisotropy with a corresponding anisotropic mesh, then the continuous analysis becomes more appropriate again, the marked discretization differences above diminish, though the bounded versus unbounded domain analyses importance remains. To conclude, for anisotropic diffusion, it is important to have optimized parameters for the discretization employed, and taking into account the subdomain sizes, especially when the anisotropy is large.

\subsection{Experiments not Covered by our Analysis}

We next study a two subdomain decomposition for DDFV discretizations where our analysis does not hold any more. We show the meshes used in Figure 6, namely a triangular mesh, a non-matching rectangular mesh, and a general polygonal mesh. We show in Table 3 the results we obtained as a function of the mesh size at the interface $h_{y}$, and we now also show the numerically optimized convergence factors $\rho_{d d f v, \text { num }}^{\text {opt }}$, and for the non-matching rectangular mesh a heuristic formula $p_{d d f v, M}^{\text {opt }}\left(\frac{h_{y}}{3}\right)$ for a theoretical value with the corresponding numerical $\rho_{d d f v, n u m}$. For the triangular mesh, we see that the discrete bounded domain analysis from Table 2 still gives quite a good prediction $p_{d d f v, M}^{o p t}$ of the numerically better performing parameter indicated by $p_{d d f v, \text { num }}^{\text {opt }}$ in Table 3 , it is just a bit too large. For the non- 


\begin{tabular}{|c|c|c|c|c|c|c|c|c|}
\hline & \multicolumn{2}{|c|}{ Triangular mesh } & \multicolumn{4}{|c|}{ non-matching rectangular mesh } & \multicolumn{2}{|c|}{ General polygonal mesh } \\
\hline$h_{y}$ & $p_{d d f v, \text { num }}^{\text {opt }}$ & $\rho_{d d f v, n u m}^{o p t}$ & $p_{d d f v, M}^{o p t}\left(\frac{h_{y}}{3}\right)$ & $\rho_{d d f v, \text { num }}$ & $p_{d d f v, \text { num }}^{o p t}$ & $\rho_{d d f v, \text { num }}^{o p t}$ & $p_{d d f v, \text { num }}^{o p t}$ & $\rho_{d d f v, n u m}^{o p t}$ \\
\hline \multicolumn{9}{|c|}{$A_{x x}=1, A_{y y}=1, \eta=1$} \\
\hline $2^{-3}$ & 7.14 & 0.36862 & 14.96 & 0.65728 & 15.48 & 0.65150 & 7.86 & 0.47536 \\
\hline $2^{-4}$ & 10.03 & 0.50431 & 21.18 & 0.74192 & 21.76 & 0.73881 & 11.52 & 0.58273 \\
\hline $2^{-5}$ & 14.02 & 0.61881 & 29.96 & 0.80624 & 29.77 & 0.80620 & 16.01 & 0.67410 \\
\hline $2^{-6}$ & 19.55 & 0.70966 & 42.37 & 0.85492 & 39.07 & 0.84919 & 22.74 & 0.74700 \\
\hline \multicolumn{9}{|c|}{$A_{x x}=16, A_{y y}=1, \eta=1$} \\
\hline $2^{-3}$ & 28.97 & 0.36842 & 62.07 & 0.55472 & 46.24 & 0.53020 & 28.74 & 0.40197 \\
\hline $2^{-4}$ & 41.01 & 0.50141 & 87.78 & 0.63339 & 74.94 & 0.58622 & 44.78 & 0.53487 \\
\hline $2^{-5}$ & 57.99 & 0.62145 & 124.15 & 0.72350 & 107.78 & 0.6909 & 61.77 & 0.63437 \\
\hline $2^{-6}$ & 78.78 & 0.71177 & 175.57 & 0.79615 & 150.21 & 0.76996 & 83.59 & 0.71457 \\
\hline \multicolumn{9}{|c|}{$A_{x x}=1, A_{y y}=16, \eta=1$} \\
\hline $2^{-3}$ & 33.89 & 0.46124 & 98.21 & 0.82432 & 110.73 & 0.81007 & 38.30 & 0.61290 \\
\hline $2^{-4}$ & 47.83 & 0.58546 & 140.66 & 0.85607 & 153.64 & 0.85031 & 57.51 & 0.68985 \\
\hline $2^{-5}$ & 67.16 & 0.68884 & 199.58 & 0.88194 & 188.27 & 0.87939 & 81.14 & 0.75575 \\
\hline $2^{-6}$ & 93.52 & 0.76320 & 282.47 & 0.91427 & 220.13 & 0.89859 & 112.62 & 0.81342 \\
\hline
\end{tabular}

Table 3: Heuristically and numerically optimized parameters $p$ for different non-conforming and nonrectangular meshes, and corresponding numerical contraction factors.

matching mesh however, it seems that the artificially cut mesh size $\frac{h_{y}}{3}$ visible in the middle in Figure 6 needs to be used in the discrete formulas to get a good prediction, both $h_{x}$ and $h_{y}$ must be divided by 3, which leads to parameters close to the best performing ones numerically. Finally, for the general polygonal last mesh, our discrete rectangular mesh analysis give rather good predictions. To conclude, the new discrete optimized parameters work also outside their scope of validity, and for non-matching grids, the smallest artificially created mesh size at the interface should be used in the theoretical formulas.

\subsection{Image Reconstruction Application}

We finally show an application of anisotropic diffusion for image reconstruction. Our domain embedded in the rectangle $(-2,2) \times(-1,1)$ is shown in Figure 7 . It represents a 2D section of a model underground, where in the refined mesh region at the bottom there is a salt dome of interest for oil recovery. On the top we have the surface of the earth, with a flat part and a downward sloped part. We decompose the domain into four subdomains, three rectangular ones and the top right one is triangular, and mesh them using different meshing techniques. In the center the decomposition has a cross point. We generate an artificial image $\tilde{u}_{0}(x, y)$ in this domain with several layers, shown in Figure 8 at the top on the left. We then add random noise to this image to obtain $u_{0}(x, y)$, which leads to the image on the top right in Figure 8. To remove the noise, one can use a time dependent diffusion equation of the form

$$
\partial_{t} u(x, y, t)=\nabla \cdot(A(x, y) \nabla u(x, y, t)), \quad u(x, y, 0)=u_{0}(x, y),
$$

and take one or a few time steps of a Backward Euler method to smooth the high frequency noise. If one does one step with the time step $\Delta t=0.1$ on the mesh in Figure 7 , and uses isotropic diffusion, $A=1$, we obtain the result shown at the bottom left of Figure 8. We see that all sharp boundaries have also been diffused, together with the noise. To avoid this, we choose now anisotropic diffusion, with

$$
A=\left(\begin{array}{cc}
1 & 0 \\
0 & 1 e-5
\end{array}\right)
$$

except in the region $-1<y<-0.75$ and $-0.3<x<0.3$ where we chose

$$
A=\left(\begin{array}{cc}
1 e-5 & 0 \\
0 & 1
\end{array}\right)
$$




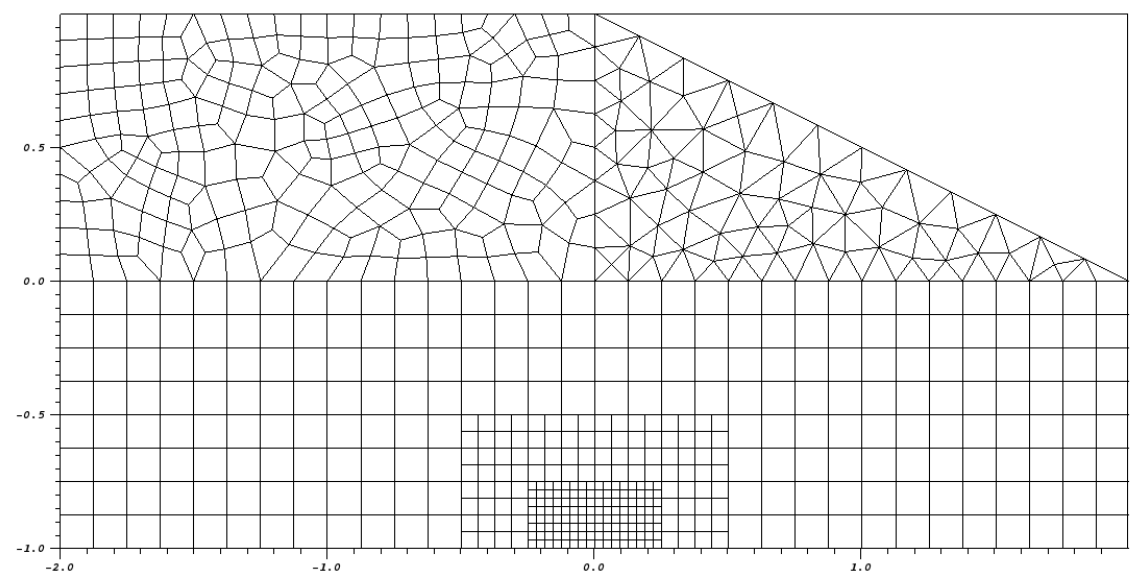

Figure 7: Domain for the image reconstruction example for a salt dome. The computations are done with a 3 times more refined mesh.
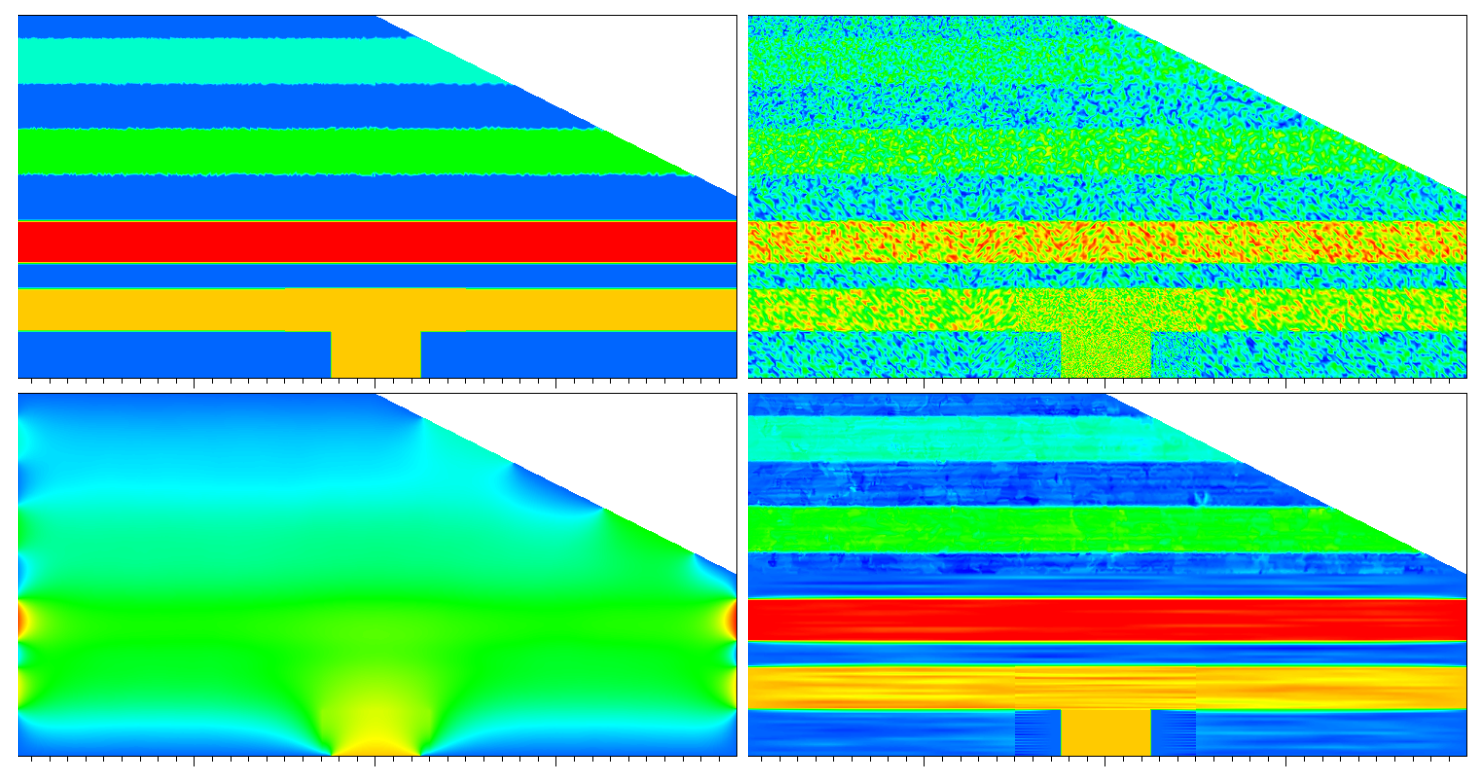

Figure 8: Top left: original image without noise. Top right: image with random noise. Bottom left: reconstructed image using isotropic diffusion. Bottom right: reconstructed image using anisotropic diffusion. 
This choice is well adapted to our image, since in the special zone, the boundaries are vertical, where in the rest they are horizontal, and we do not want to diffuse across the sharp interfaces, only along them. The reconstruction computed with our algorithm is shown at the bottom right in Figure 8. We clearly see that anisotropic diffusion is very well capable of preserving sharp edges in the noise removal process. In a more realistic situation one would need to use in the anisotropic diffusion tensor the gradient of $u$ to determine the diffusion directions to avoid. Using the Robin parameter $p=0.05$, our algorithm needed 14 iterations to converge to a tolerance of $1 e-3$, whereas with $p=0.01$ it took 45 iterations, and with $p=1.5$ it took 213 iterations. This illustrates well the importance of a good choice of the parameter $p$.

\section{Conclusion}

Discrete Duality Finite Volume methods (DDFV) are a recent class of powerful discretizations for anisotropic diffusion problems. They reach high accuracy also on distorted meshes and for high anisotropies due to excellent gradient reconstructions. DDFV methods require however more unknowns than classical finite volume methods, and therefore good solvers are needed. Optimized Schwarz methods (OSM) are excellent candidates for this task, since they are naturally parallel, and their performance can also be tuned for highly anisotropic diffusion. We proved convergence of a non-overlapping Schwarz method with Robin transmission conditions for a very general decomposition of a DDFV discretized anisotropic diffusion problem into many subdomains including cross points, and also discovered that the Robin parameter can be optimized separately for primal and dual grid components in DDFV. We derived such asymptotically optimized parameters that are easy to use in practice, and showed in numerical experiments that very good convergence speeds are achieved, also for the concrete application of image reconstruction. This application shows however also a further research direction, namely how the optimized parameters should be adapted to highly variable coefficients, a case not covered by the present analysis. Also higher order transmission conditions of Ventcell type should be investigated which can further accelerate OSMs. Finally, one should also study the preconditioning capabilities of our OSMs for Krylov methods, but these topics will be addressed elsewhere.

\section{References}

[1] B. Andreianov, F. Boyer, and F. Hubert. Discrete duality finite volume schemes for Leray-Lions type elliptic problems on general 2D-meshes. Num. Meth. for PDEs, 23(1):145-195, 2007.

[2] P.-M. Berthe, C. Japhet, and P. Omnes. Space-time domain decomposition with finite volumes for porous media applications. In Domain decomposition methods in science and engineering XXI, pages 567-575. Springer, 2014.

[3] F. Boyer and F. Hubert. Finite volume method for 2d linear and nonlinear elliptic problems with discontinuities. SIAM J. Numer. Anal., 46, 2008.

[4] F. Boyer, F. Hubert, and S. Krell. Nonoverlapping Schwarz algorithm for solving two-dimensional m-DDFV schemes. IMA journal of numerical analysis, 30(4):1062-1100, 2010.

[5] Y. Coudière and G. Manzini. The discrete duality finite volume method for convection-diffusion problems. SIAM Journal on Numerical Analysis, 47(6):4163-4192, 2010. Available online at http://dx.doi.org/10.1137/080731219.

[6] S. Delcourte, K. Domelevo, and P. Omnes. A discrete duality finite volume approach to Hodge decomposition and div-curl problems on almost arbitrary two-dimensional meshes. SIAM J. Numer. Anal., 45(3):1142-1174 (electronic), 2007.

[7] S. Delcourte and P. Omnes. A Discrete Duality Finite Volume discretization of the vorticity-velocitypressure formulation of the 2D Stokes problem on almost arbitrary two-dimensional grids. Numer. Methods PDEs, pages 1-30, 2015. 
[8] B. Després. Méthodes de décomposition de domaine pour la propagation d'ondes en régime harmonique. PhD thesis, Université Dauphine, Paris, 1991.

[9] K. Domelevo and P. Omnes. A finite volume method for the Laplace equation on almost arbitrary two-dimensional grids. M2AN Math. Model. Numer. Anal., 39(6):1203-1249, 2005.

[10] O. Drblíková and K. Mikula. Convergence analysis of finite volume scheme for nonlinear tensor anisotropic diffusion in image processing. SIAM Journal on Numerical Analysis, 46(1):37-60, 2007.

[11] Y. Fukuda, I. Ohashi, K. Hanafusa, T. Nakagawa, S.-i. Ohtani, Y. An-naka, T. Hayashi, and H. Shibuya. Anisotropic diffusion in kidney: apparent diffusion coefficient measurements for clinical use. Journal of Magnetic Resonance Imaging: An Official Journal of the International Society for Magnetic Resonance in Medicine, 11(2):156-160, 2000.

[12] M. J. Gander. Optimized Schwarz method. SIAM Journal on Numerical Analysis, 44(2):699-731, 2006.

[13] M. J. Gander. Schwarz methods over the course of time. Electron. Trans. Numer. Anal, 31(5):228$255,2008$.

[14] M. J. Gander. On the influence of geometry on optimized Schwarz methods. SeMA Journal, 53(1):7178, 2011.

[15] M. J. Gander and O. Dubois. Optimized Schwarz methods for a diffusion problem with discontinuous coefficient. Numerical Algorithms, 69(1):109-144, 2015.

[16] M. J. Gander, L. Halpern, F. Hubert, and S. Krell. DDFV Ventcell Schwarz algorithms. In Domain Decomposition Methods in Science and Engineering XXII, pages 481-489. Springer, 2016.

[17] M. J. Gander, L. Halpern, F. Hubert, and S. Krell. Optimized schwarz methods with general ventcell transmission conditions for fully anisotropic diffusion with discrete duality finite volume discretizations. Moroccan Journal of Pure and Applied Analysis, 2(7):182-213, 2021.

[18] M. J. Gander, F. Hubert, and S. Krell. Optimized Schwarz algorithms in the framework of ddfv schemes. In Domain Decomposition Methods in Science and Engineering XXI, pages 457-466. Springer, 2014.

[19] M. J. Gander and F. Kwok. Best Robin parameters for optimized Schwarz methods at cross points. SIAM Journal on Scientific Computing, 34(4):A1849-A1879, 2012.

[20] M. J. Gander and K. Santugini. Cross-points in domain decomposition methods with a finite element discretization. Electronic Transactions on Numerical Analysis, 45:219-240, 2016.

[21] N. Hartung and F. Hubert. An efficient implementation of a 3D CeVeFE DDFV scheme on cartesian meshes and an application in image processing. In Proceedings of the 7 th conference on Finite Volumes and Complex Applications, Berlin, June 2014.

[22] R. D. Haynes and K. Mohammad. Fully discrete Schwarz waveform relaxation on two bounded overlapping subdomains. In Domain Decomposition Methods in Science and Engineering XXV, LNCSE. Springer-Verlag, 2020.

[23] F. Hermeline. Approximation of diffusion operators with discontinuous tensor coefficients on distorted meshes. Comput. Methods Appl. Mech. Engrg., 192(16-18):1939-1959, 2003.

[24] F. Hermeline, S. Layouni, and P. Omnes. A finite volume method for the approximation of Maxwell's equations in two space dimensions on arbitrary meshes. J. Comput. Phys., 227(22):9365-9388, 2008.

[25] J. Kačur and K. Mikula. Solution of nonlinear diffusion appearing in image smoothing and edge detection. Applied Numerical Mathematics, 17(1):47-59, 1995. 
[26] S. Krell. Schémas Volumes Finis en mécanique des fluides complexes. PhD thesis, Université de Provence-Aix-Marseille I, 2010.

[27] P. L. Lions. On the Schwarz alternating method. III. A variant for nonoverlapping subdomains. In Third International Symposium on Domain Decomposition Methods for Partial Differential Equations (Houston, TX, 1989), pages 202-223. SIAM, Philadelphia, PA, 1990.

[28] S. Loisel, J. Côté, M. J. Gander, L. Laayouni, and A. Qaddouri. Optimized domain decomposition methods for the spherical Laplacian. SIAM Journal on Numerical Analysis, 48(2):524-551, 2010.

[29] K. Mikula and N. Ramarosy. Semi-implicit finite volume scheme for solving nonlinear diffusion equations in image processing. Numerische Mathematik, 89(3):561-590, 2001.

[30] P. Perona and J. Malik. Scale-space and edge detection using anisotropic diffusion. IEEE Transactions on pattern analysis and machine intelligence, 12(7):629-639, 1990.

[31] L. R. Van Loon, J. M. Soler, W. Müller, and M. H. Bradbury. Anisotropic diffusion in layered argillaceous rocks: a case study with opalinus clay. Environmental science $\mathscr{E}$ technology, 38(21):5721$5728,2004$.

[32] J. Weickert. Anisotropic diffusion in image processing, volume 1. Teubner Stuttgart, 1998.

[33] S.-L. Wu and M. D. Al-Khaleel. Semi-discrete Schwarz waveform relaxation algorithms for reaction diffusion equations. BIT Numerical Mathematics, 54(3):831-866, 2014.

[34] S.-L. Wu and M. D. Al-Khaleel. Optimized waveform relaxation methods for RC circuits: discrete case. ESAIM: Mathematical Modelling and Numerical Analysis, 51(1):209-223, 2017. 